\title{
4
}

\section{Ultrasound Evaluation of Peripheral Nerves}

Ultrasound evaluation of peripheral nerves and peripheral nerve pathology began being described in the late 1980s with the development of higher frequency transducers (Fornage, 1988). As the instrumentation improved with advances in technology that led to higher resolution, ultrasound became the modality of choice for assessing most peripheral nerves that are not obscured by bone (Bacigalupo et al., 2003; Bianchi, 2008; Fornage, 1993).

Ultrasound can be used for diagnosis of peripheral nerve disorders related to compression neuropathies, trauma, and tumors (Koenig et al., 2009). In some cases, it can provide information about the type and severity of the lesion (Tagliafico et al., 2010). It is also an ideal modality for performing peripheral nerve blocks. Successful sonographic evaluation of peripheral nerves in a clinical setting requires an indepth knowledge of peripheral nerve anatomy and appearance, scanning and measurement techniques, and changes in pathologic states.

\section{EVALUATION OF PERIPHERAL NERVE}

\section{NORMAL NERVE}

\section{Anatomy}

The normal nerve displays an uninterrupted fascicular or filamentous pattern, unlike the intercalated pattern of a typical tendon (Chiou et al., 2003). Nerve fascicles are not as tightly packed as the fibers in a tendon (Beekman and Visser, 2004; FIGURE 4-1). Nerves have an epineurium, which is the external sheath that surrounds the nerve fascicles. Epineurium is seen as a bright signal on ultrasound, and the nerve fascicles are dark (FIGURES 4-2 and 4-3). The fascicles are enveloped by the perineurium. The individual fibers are enveloped by the endoneurium. The tissue between the fascicles and the outer sheath is sometimes referred to as the "inner epineurium" and the external sheath is referred to as the "outer epineurium."

Peripheral nerves have an extensive vascular supply to provide for their metabolic needs. Large peripheral nerves are often accompanied by arteries and veins that are easily recognized with ultrasound. Nerves also have a network of vessels that travel longitudinally in their epineurium with branches that enter as endoneural vessels among the fascicles (Bianchi and Martinoli, 2007; FIGURE 4-4).

Peripheral nerves generally display a characteristic appearance with high-frequency ultrasonography. In short-axis view, nerves display a "honeycomb" appearance with hypoechoic fascicles lying inside a more hyperechoic background (Silvestri et al., 1995; FIGURE 4-5). In long-axis view, peripheral nerves display a characteristic fascicular pattern within multiple longitudinal hypoechoic bands of fascicular bundles. The hyperechoic bands are the intervening 


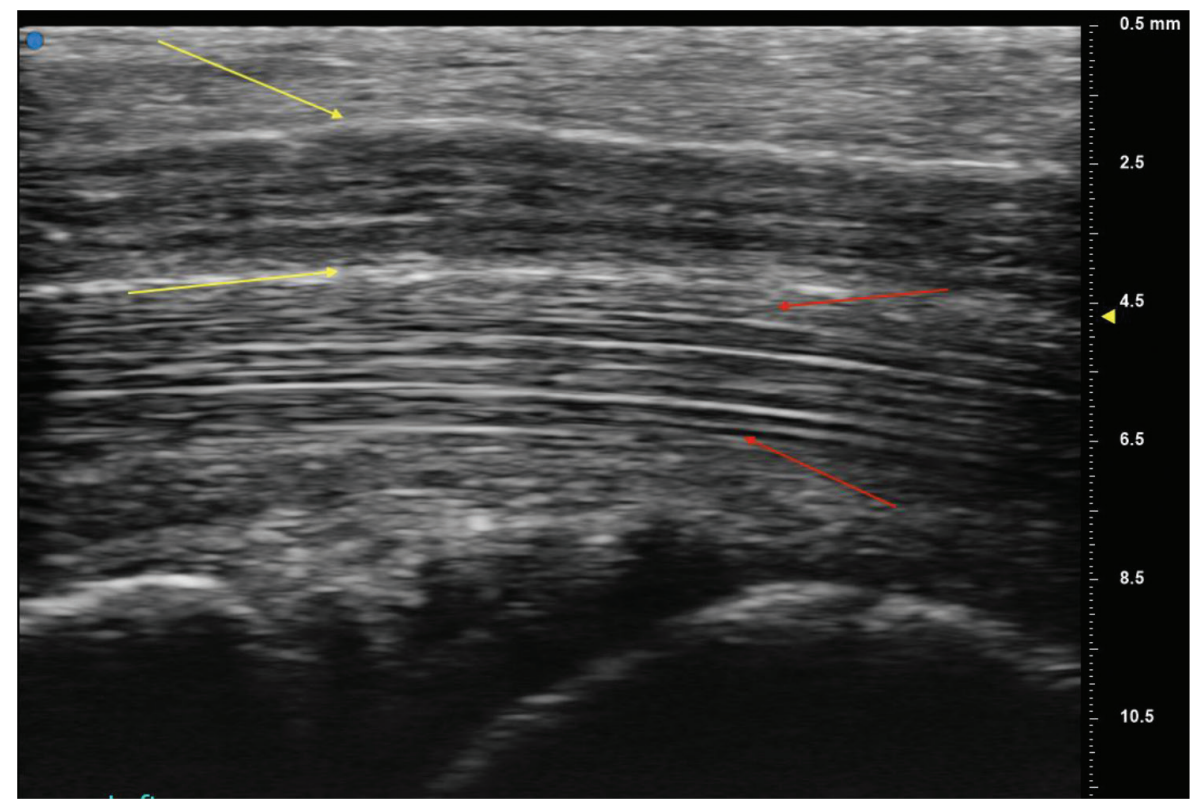

(A)

\section{FIGURE 4-1}

Sonograms of peripheral nerve and tendon. (A) The long-axis view of the nerve (between the yellow arrows) displays the large hypoechoic fascicles. The tendon (between the red arrows) displays the intercalated fibrillar pattern. (B) The short-axis view of the nerve (yellow arrow) and tendons (red arrows). The cross section of the fascicular pattern has the appearance of a honeycomb. The cross section of the finer fibrillar pattern appears like the cut end of a broom.

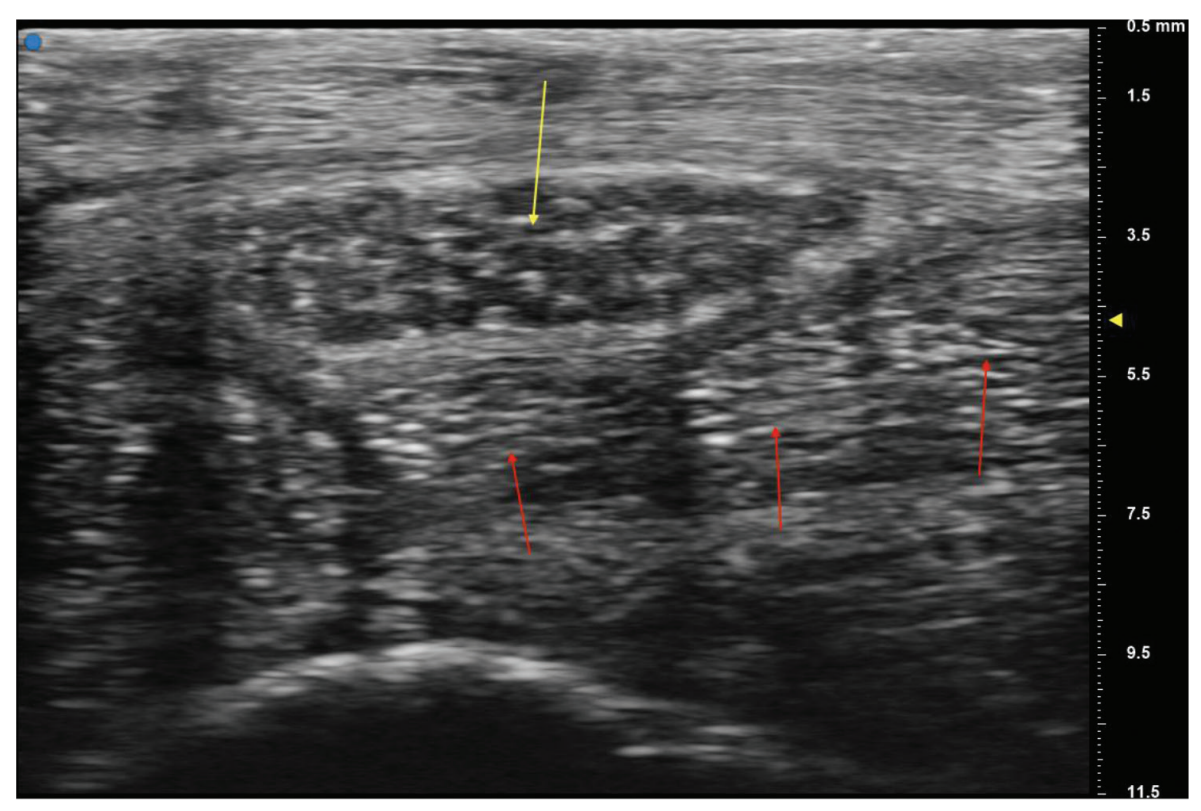

(B)

epineurium. The size and number of fascicles seen in nerves can be highly variable depending on the nerve size and its location in the body (Peer and Bodner, 2008). Short-axis view of the nerve is generally the best image to differentiate it from other surrounding tissue. It also assists in understanding the relationship to adjacent structures. Longitudinal views provide valuable information but are more difficult to obtain as they frequently curve out of the field of view (FIGURE 4-6).

\section{Imaging Strategies}

When it is not clear whether the structure being imaged is a nerve, the examiner should make an effort to follow its course both proximally and distally (Créteur et al., 2007). This can often result in moving from an area that is more isoechoic, such as tendon, to an area that is more hypoechoic, such as muscle, where the nerve is much easier to distinguish (FIGURE 4-7; VIDEO 4-1). Toggling the transducer can 
also help distinguish the nerve from surrounding tis- sue that has more anisotropy (FIGURE 4-8; VIDEO 4-2). As long as the ultrasound instrument has a good resolution at faster frame rates, scanning more rapidly often creates a greater distinction between nerve and surrounding tissue than scanning more slowly

- (VIDEOS 4-3 and 4-4). Using a long pathway of coupling gel along the direction of scanning can assist with rapid evaluation (FIGURE 4-9). Becoming familiar with characteristic bony acoustic and soft tissue

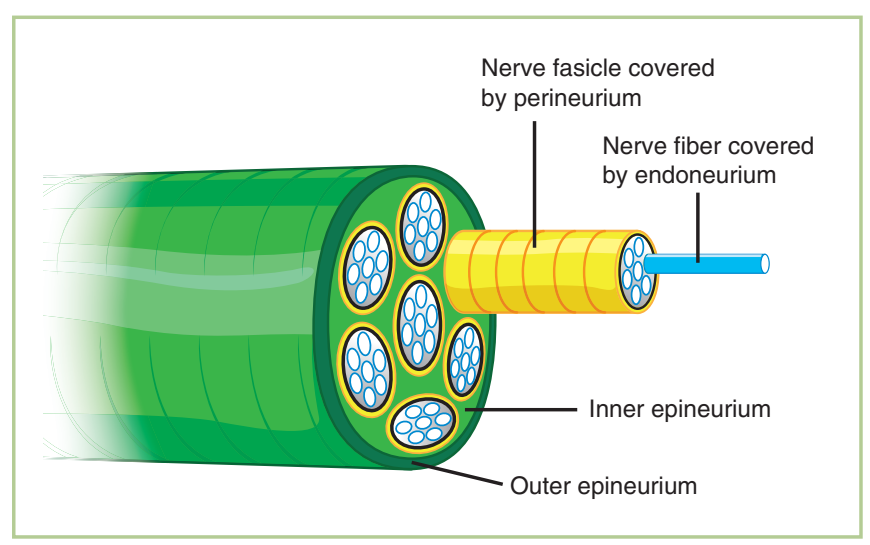

\section{FIGURE 4-2}

A nerve cross section. The endoneurium covers the nerve fiber. Bundles of nerve fibers make a nerve fascicle, which is covered by perineurium. The epineurium covers the bundles of fascicles. landmarks can help identify various nerves (FIGURE 4-10). This includes characteristic vessels.

Attention to the pressure on the transducer is needed when scanning peripheral nerves. The soft tissue around the nerve as well as the nerve itself transforms with excessive transducer pressure (FIGURE 4-11). Transformation of the nerve shape is particularly evident with hard pressure when the nerve is against a hard bony structure. The shape change is a result of relative flexibility of the epineurium. The perineurium is relatively inflexible and, therefore, the fascicles do not deform with routine pressure but will redistribute inside the shape-altered epineurium. If there is focal change in the nerve shape despite only light pressure, or if there are alterations in the shape of the fascicles, then this should be considered an abnormality (Martinoli et al., 2000). Attention should be given to the amount of transducer pressure on the tissue when assessing for shape changes of the nerve. This is particularly important when performing calculations such as flattening ratios.

Intentional variation of transducer pressure can be used in some areas to increase the conspicuity of both the nerve and the surrounding tissue. With high frequencies, the compression can sometimes increase the resolution of the tissue. Conversely, an increased through-transmission of the sound waves can improve the visualization of nerves lying deep to veins or cystic structures. This can potentially be affected by compression (FIGURE 4-12; VIDEO 4-5).

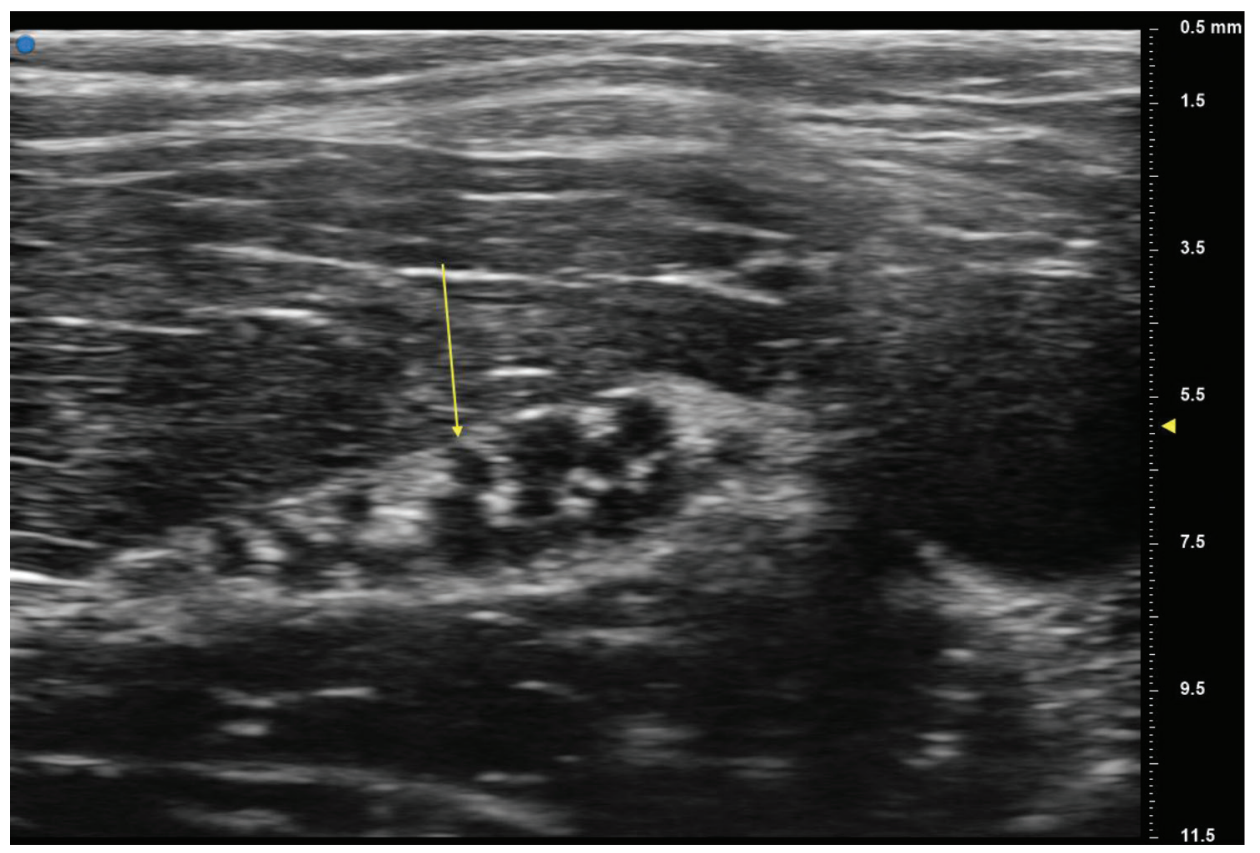

FIGURE 4-3

Sonogram of the short-axis architecture of the proximal median nerve (yellow arrow). Note the hypoechoic fascicles surrounded by the hyperechoic epineurium. 


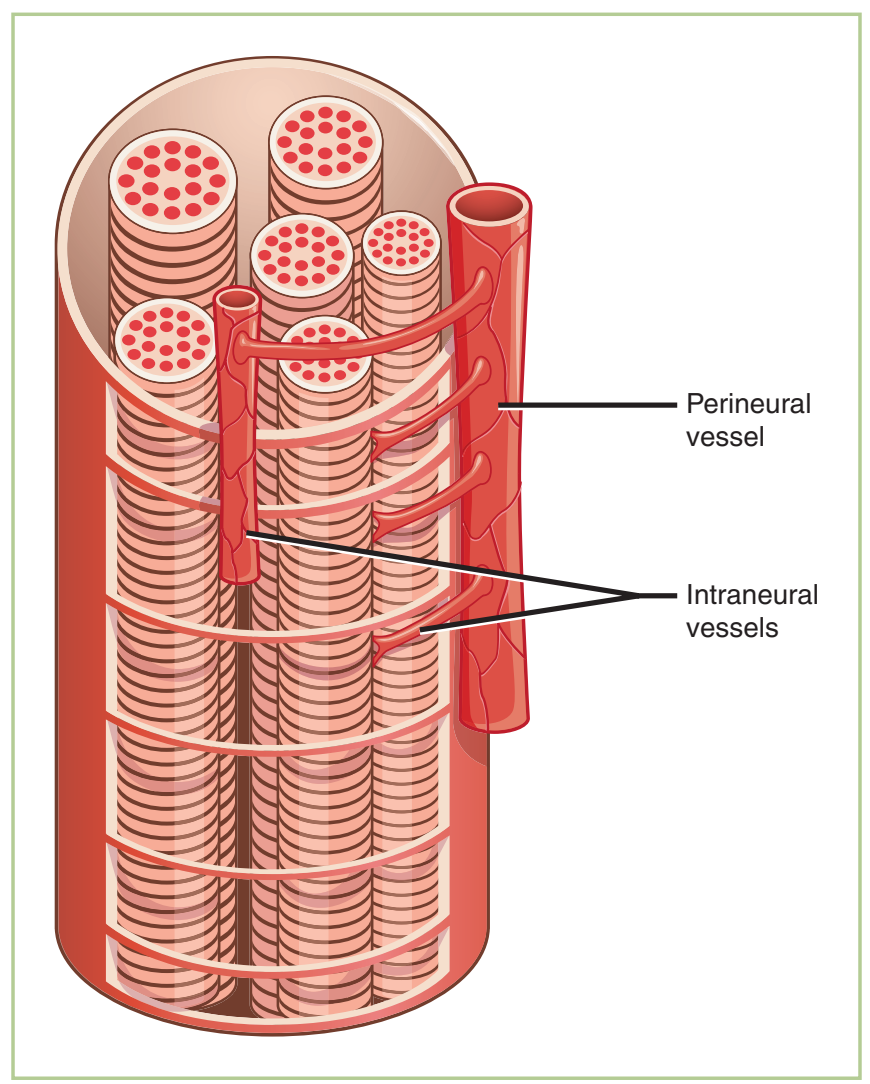

FIGURE 4-4

The vascular supply of a peripheral nerve. The perineural vessels run alongside the nerve and the intraneural vessels pierce the epineurium and run alongside the fascicles.
Anatomic variations exist in peripheral nerves, and ultrasound can be used to identify these. Knowledge of common anatomic variations is essential so that they are not confused with pathologic conditions. An example of a frequently cited variation is the proximal bifurcation of the median nerve creating a bifid appearance (Iannicelli et al., 2000; FIGURE 4-13).

The effect of transducer frequency on sonographic images is discussed in detail in Chapter 3. In general, the highest frequency transducer that will allow sufficient depth of sound wave penetration should be used for optimum resolution of peripheral nerves. Conversely, also inspecting the area around the nerve at lower frequencies can sometimes provide enhanced conspicuity of the surrounding tissue (FIGURE 4-14).

\section{Nerve Measurement}

The use of perimeter tracing to determine the precise cross-sectional area has been shown to be a reliable technique that correlates with cadaver measurements. The cross-sectional area of nerves in short axis can be obtained by tracing with an electronic caliper around the margin of the hypoechoic nerve fascicles and inside the hyperechoic outer epineurium (Nakamichi and Tachibana, 2002; FIGURE 4-15). Nerve size generally decreases when imaged from proximally to

\section{FIGURE 4-5}

Sonogram of the sciatic nerve (yellow arrow) in short axis demonstrating the "honeycomb" pattern representing the hypoechoic fascicles between the hyperechoic epineurium.

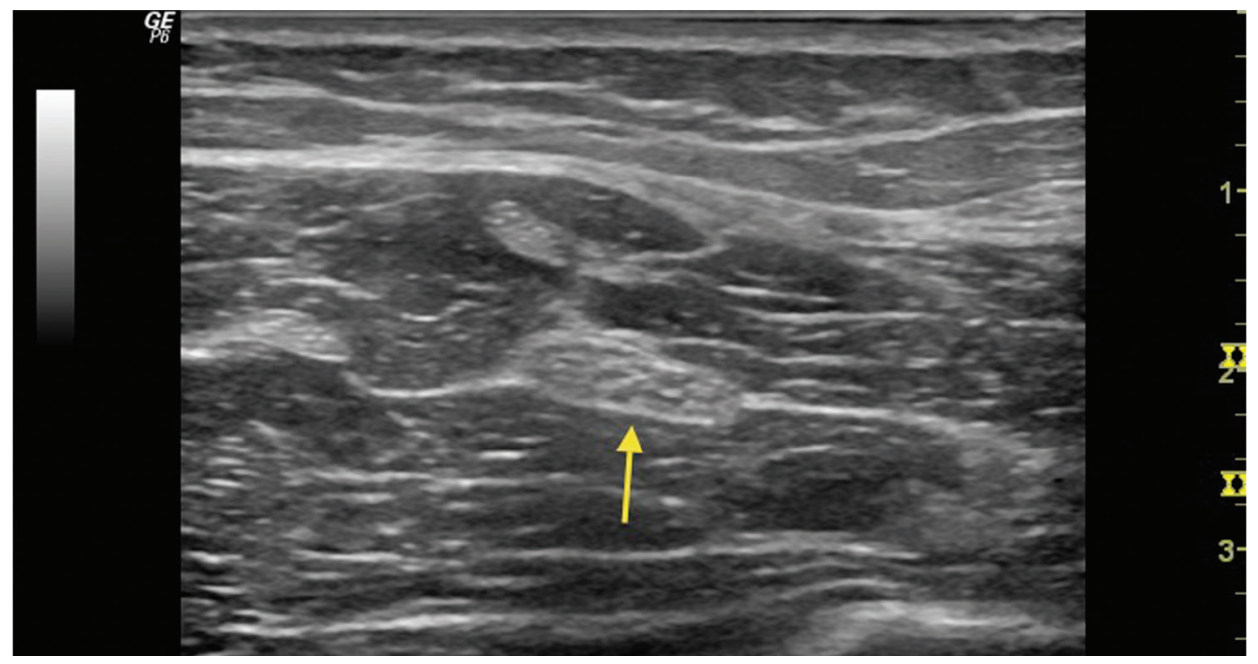



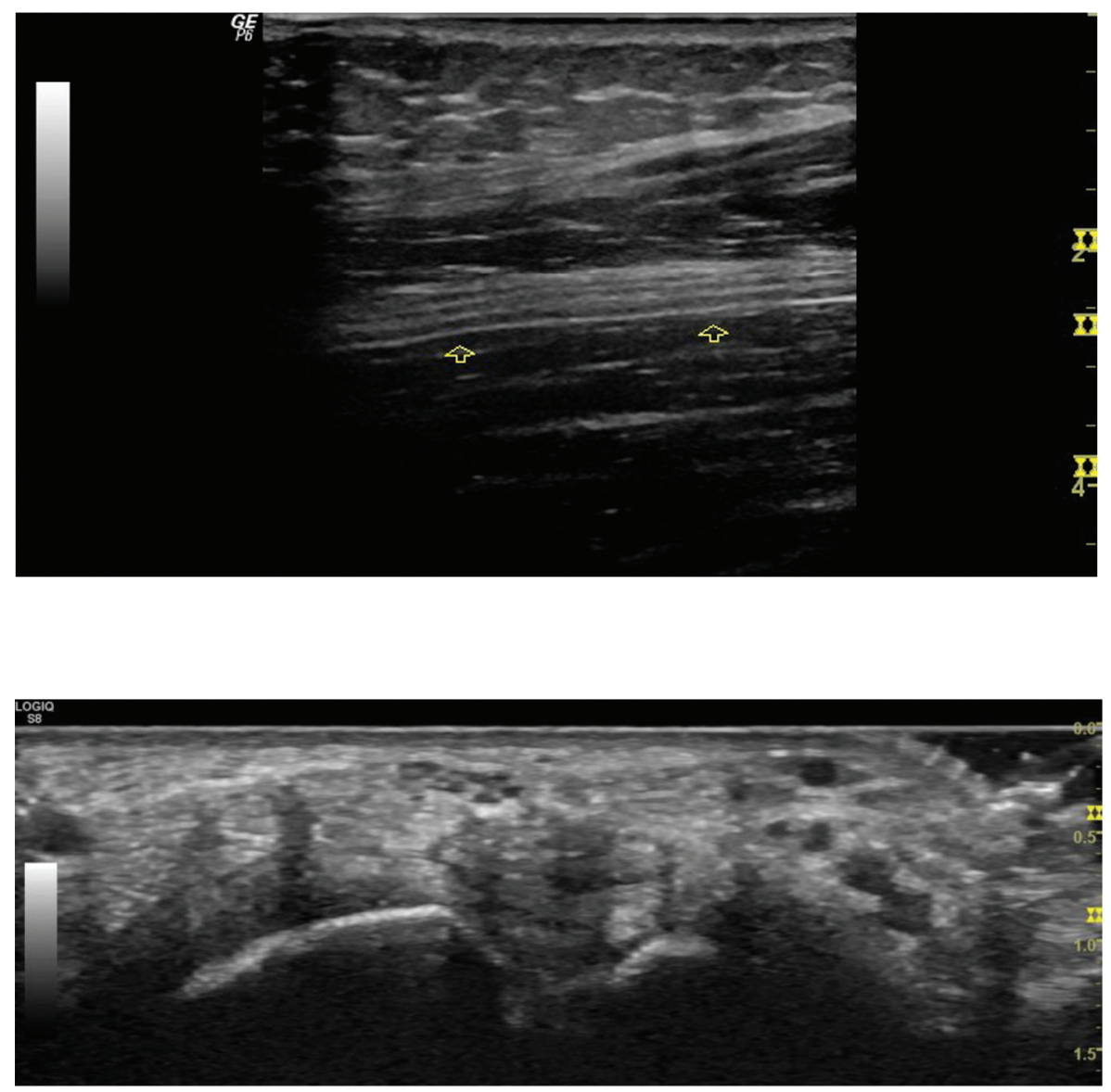

(A)

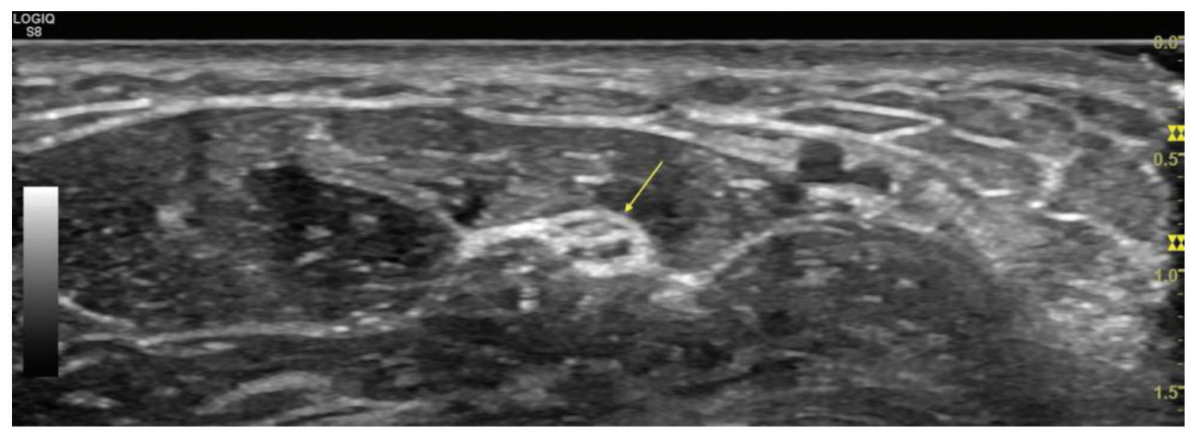

(B)

\section{FIGURE 4-6}

Sonogram of the sciatic nerve in long axis (yellow arrowheads) demonstrating the characteristic fascicular pattern of a peripheral nerve.

\section{FIGURE 4-7}

Sonograms demonstrating the benefit of following the nerve to a more conspicuous area when it is difficult to see. (A) In this case, the median nerve is more difficult to visualize in short-axis view at the wrist around the relatively isoechoic tendons. (B) The median nerve (yellow arrow) is more conspicuous in the region more proximally where it is surrounded by more hypoechoic muscle. Note the brighter appearance of the outer epineurium. Once reliably identified, the nerve can be traced to the original location. Following a nerve in this fashion can assist in localization.

distally as the branches leave the main nerve trunk. The cross-sectional area of nerves tends to be larger in taller individuals (Zaidman et al., 2009) and smaller in women (Cartwright, Shin et al., 2007).

Nerve swelling can be measured reliably by most instruments. It is critical that the transducer be placed perpendicular to the nerve for accuracy. Measurement should be performed from the inner border of the echogenic epineurium surrounding the fascicles for consistency (FIGURE 4-16). This is generally preferred over measurement of the outer border. The outer portion of the epineurium can be more 


\section{FIGURE 4-8}

Sonograms of the effect of toggling the angle of the transducer to produce anisotropic effect on the surrounding tendons (red arrows), making the peripheral nerve (yellow arrow) more conspicuous. (A) The angle of the sound beam is orthogonal to the structures, resulting in less contrast between them. (B) The angle is changed, causing more anisotropic artifact of the tendons, which results in a more conspicuous nerve.

\section{FIGURE 4-9}

The use of a long pathway of coupling gel to facilitate rapid scanning of the nerve.

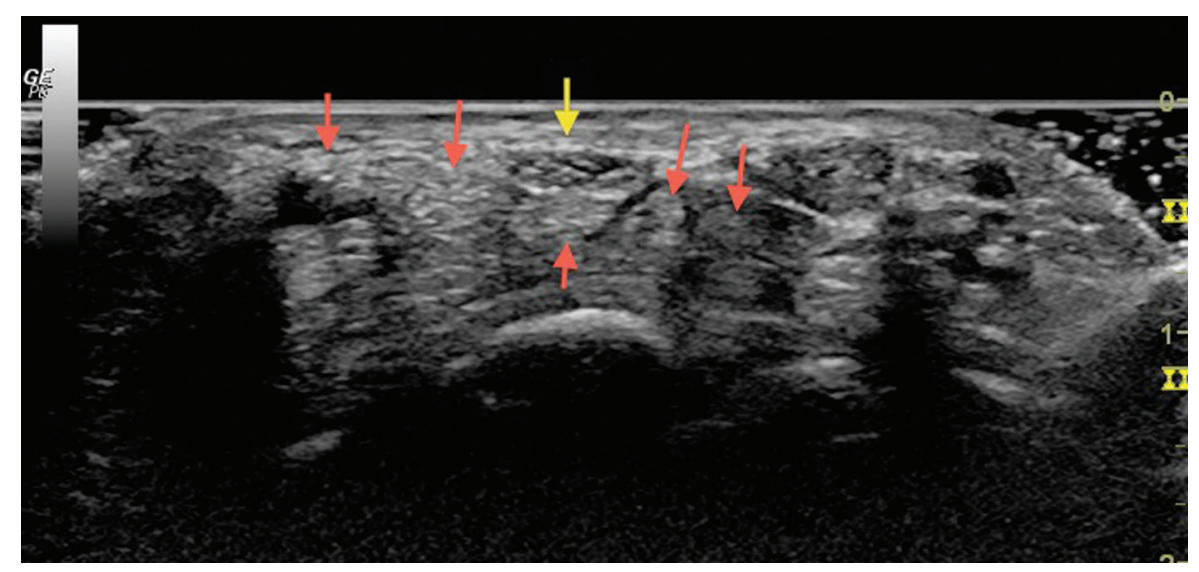

(A)

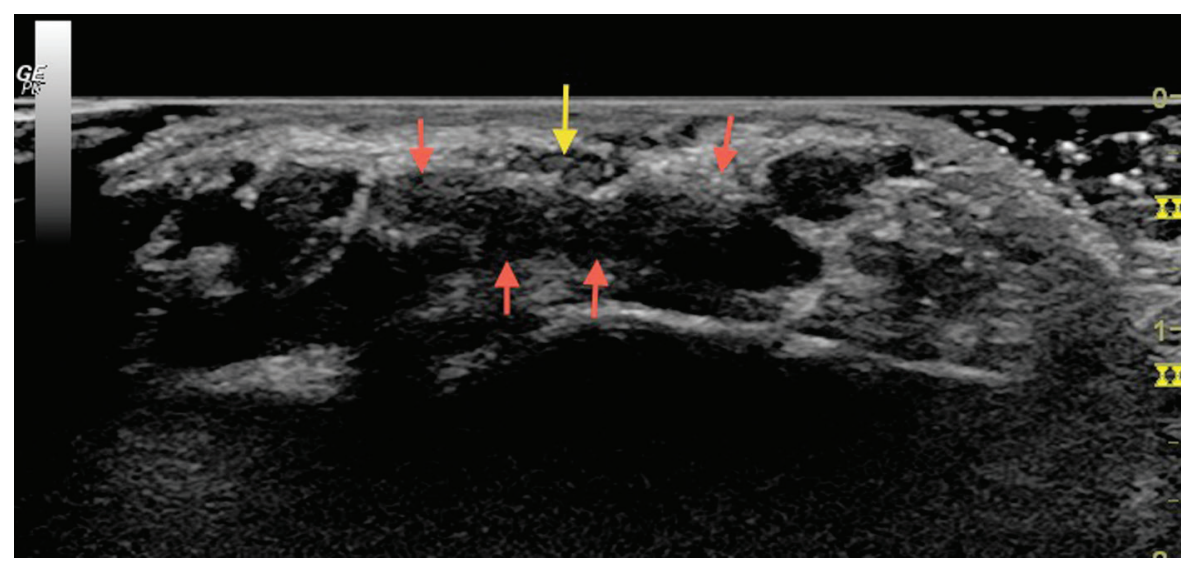

(B)

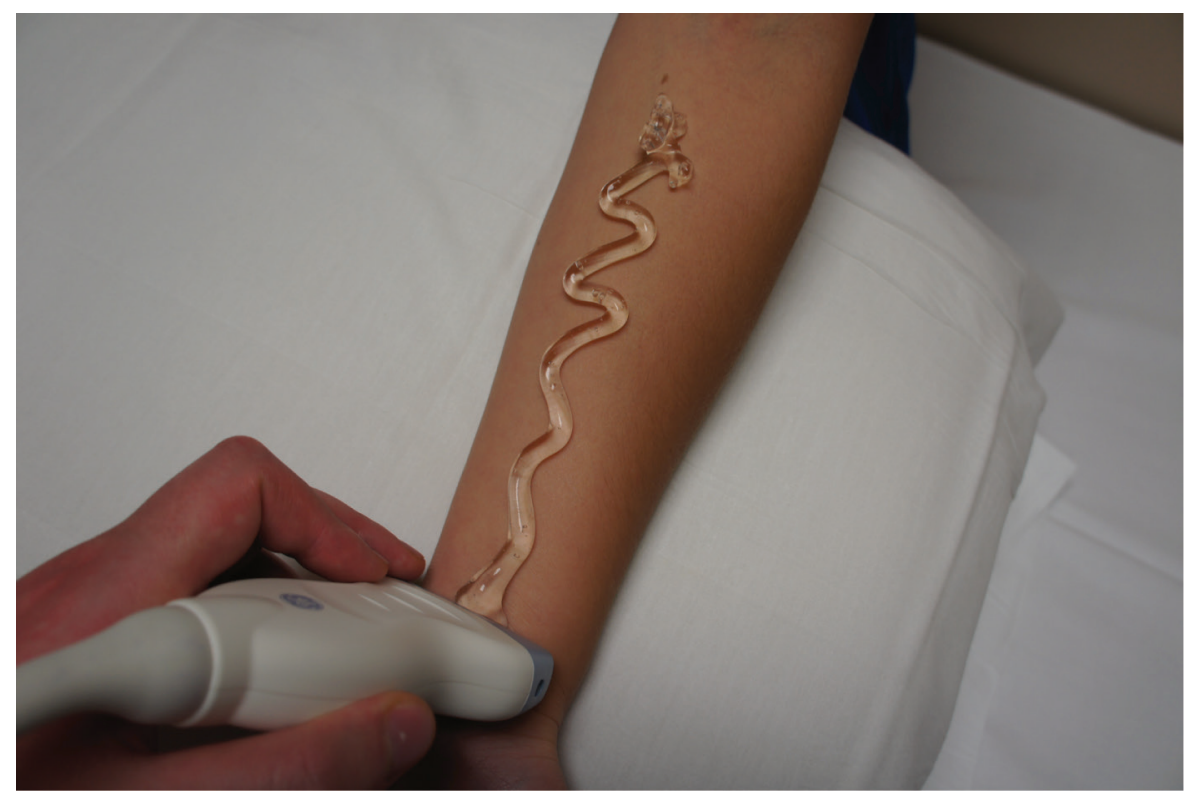




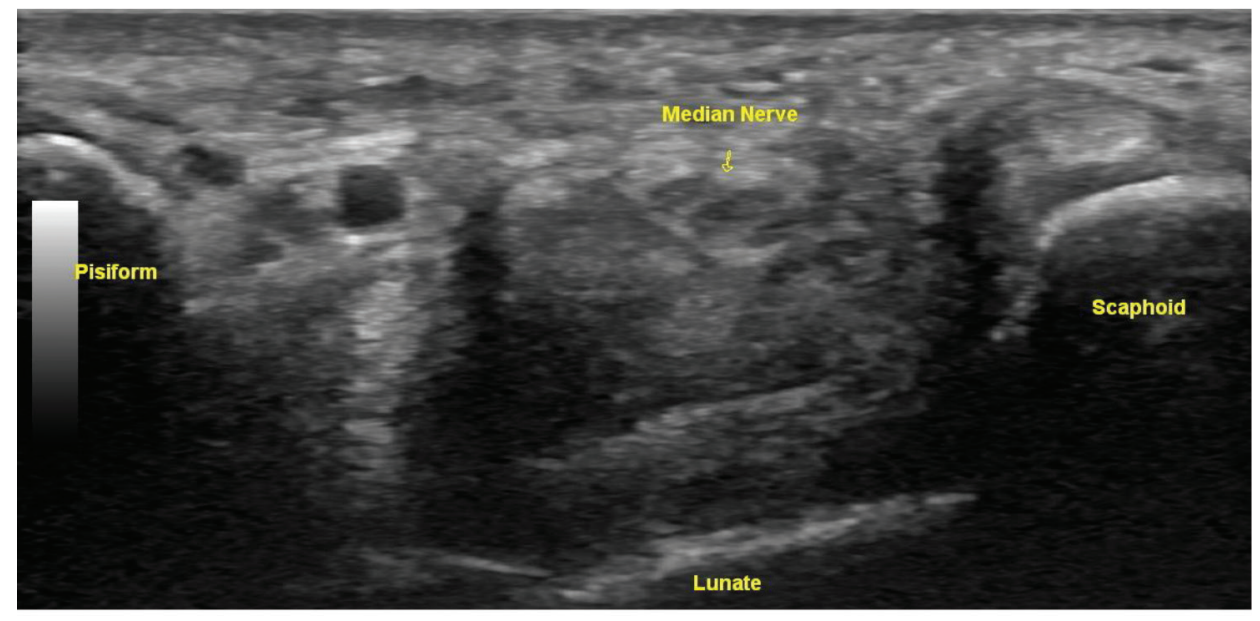

(A)

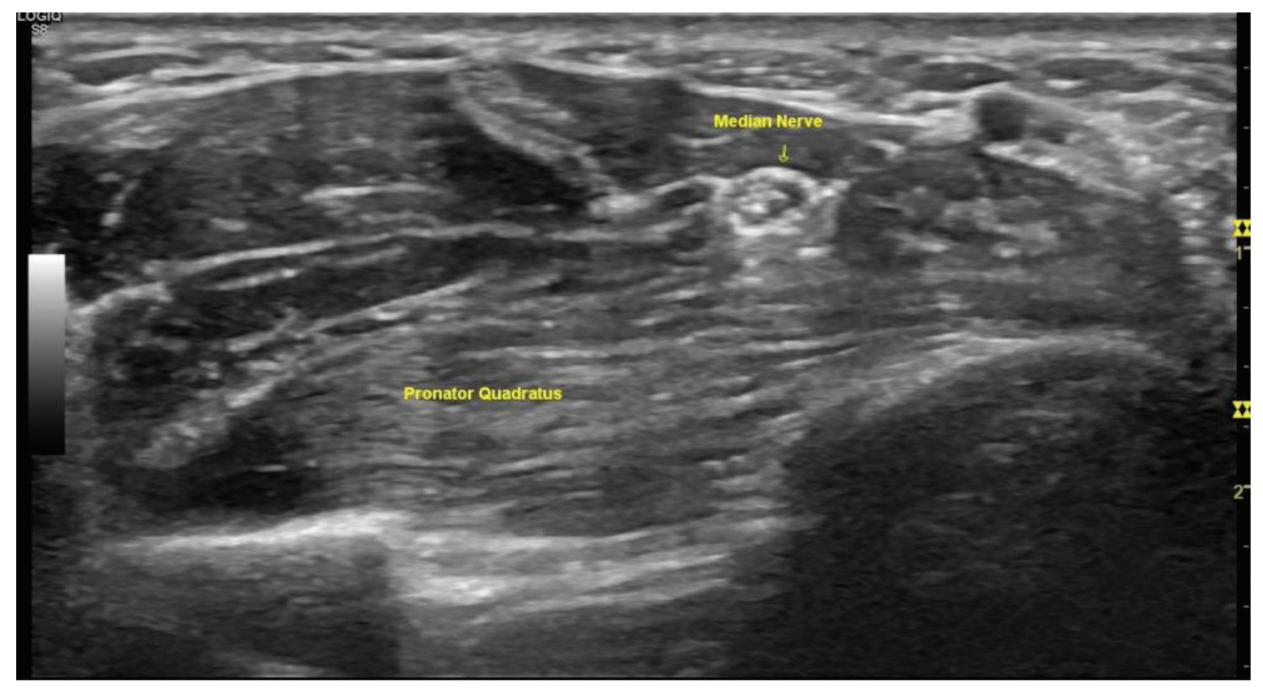

(B)

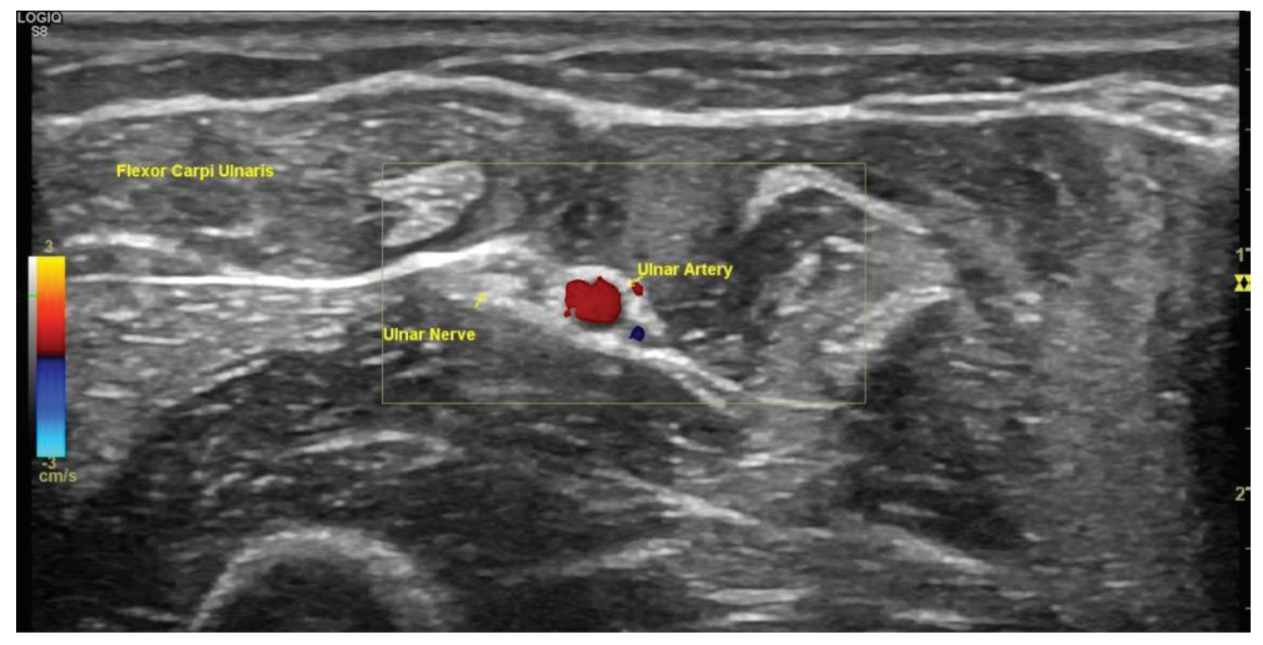

(C)

\section{FIGURE 4-10}

Examples of characteristic landmarks that can be used to identify specific nerves in short axis at specific locations. (A) The median nerve at the carpal tunnel inlet identified by the bony acoustic landmarks of the pisiform, lunate, and scaphoid. (B) The median nerve at its location in the distal forearm, indicated by the long-axis muscle pattern of the pronator quadratus. (C) The location of the ulnar nerve at the mid-forearm noted by the ulnar artery and the overlying position of the flexor carpi ulnaris (FCU). (continued) 


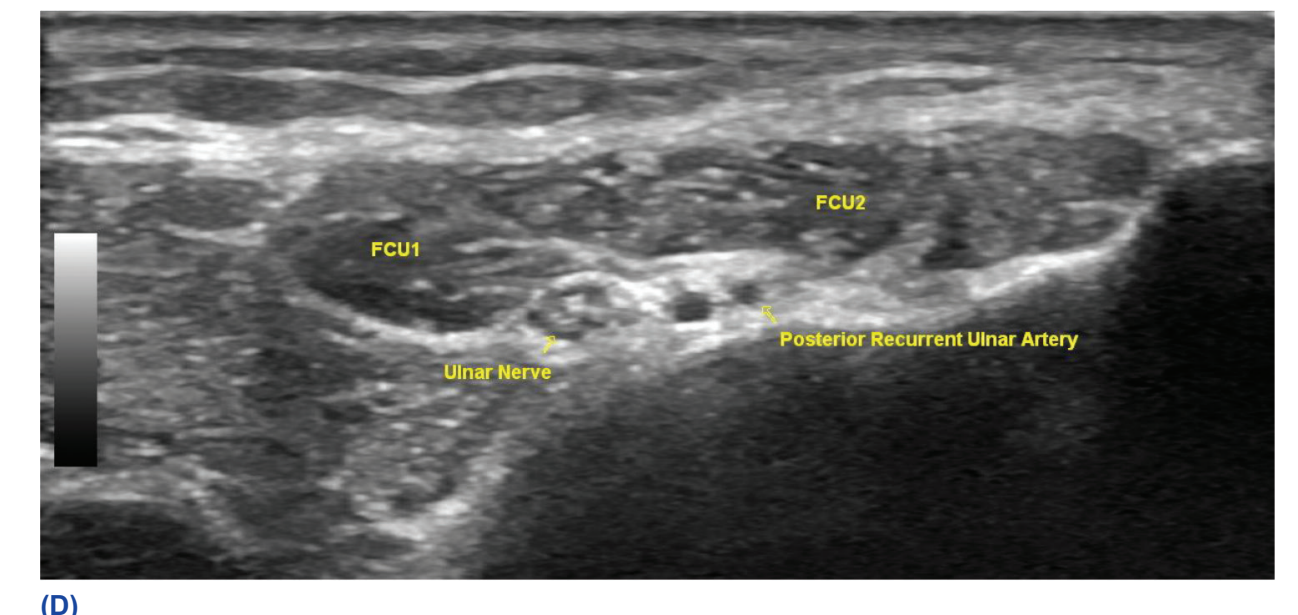

(D)

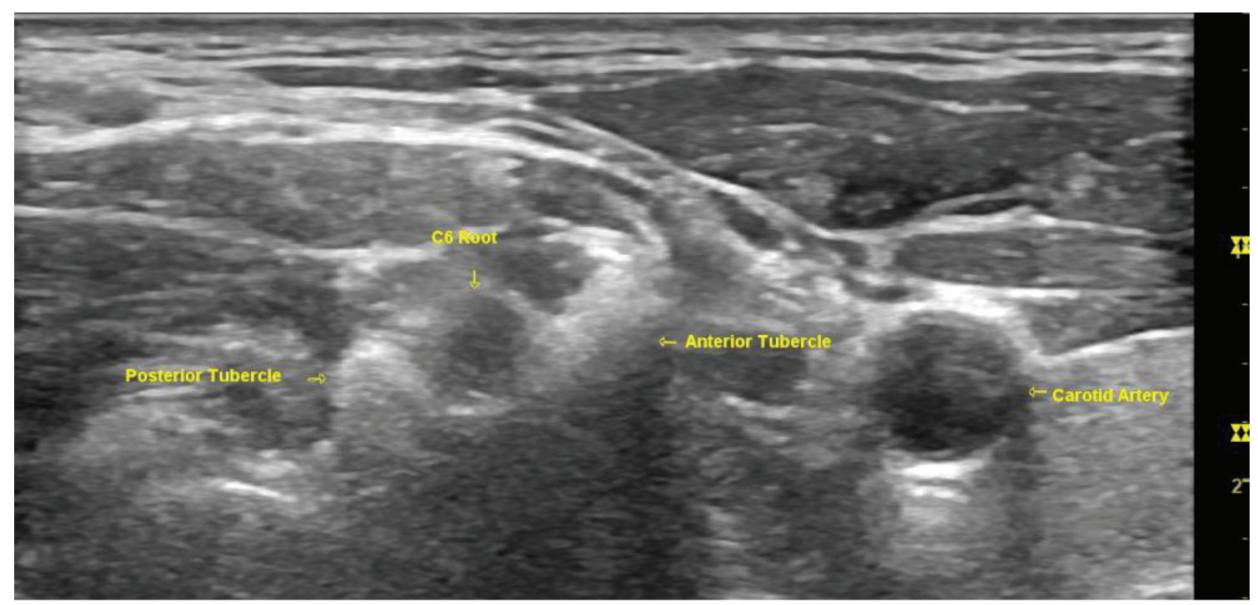

(E)

FIGURE 4-10 (Continued)

(D) The ulnar nerve at the cubital tunnel. FCU 1 and 2 denote the two origins of the flexor carpi ulnaris. (E) The C6 nerve root lying between the anterior tubercle and posterior tubercle of the transverse spinous process. (F) The fibular nerve at the fibular head. (continued)

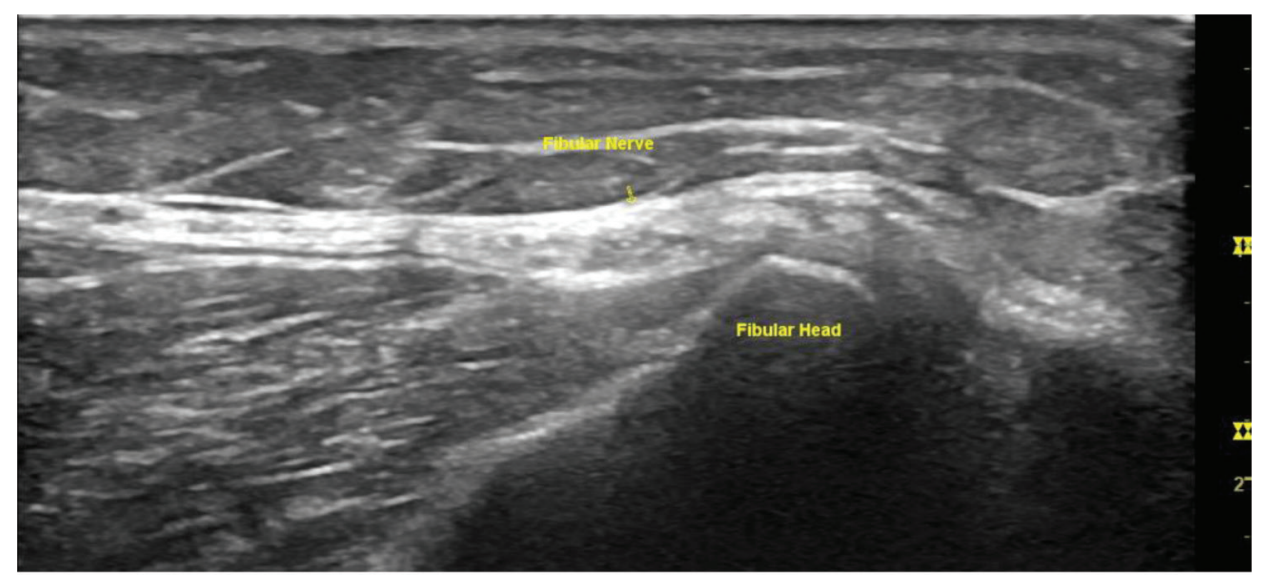

(F) 


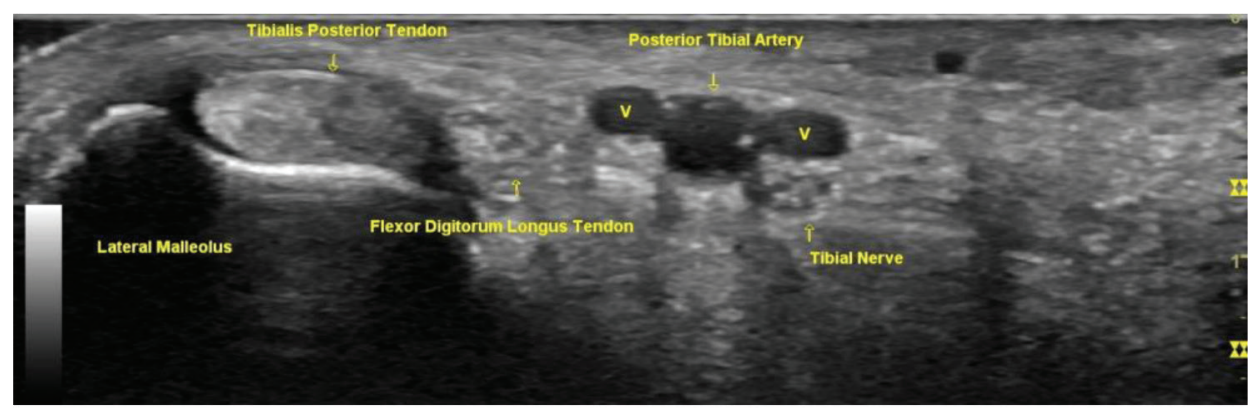

(G)

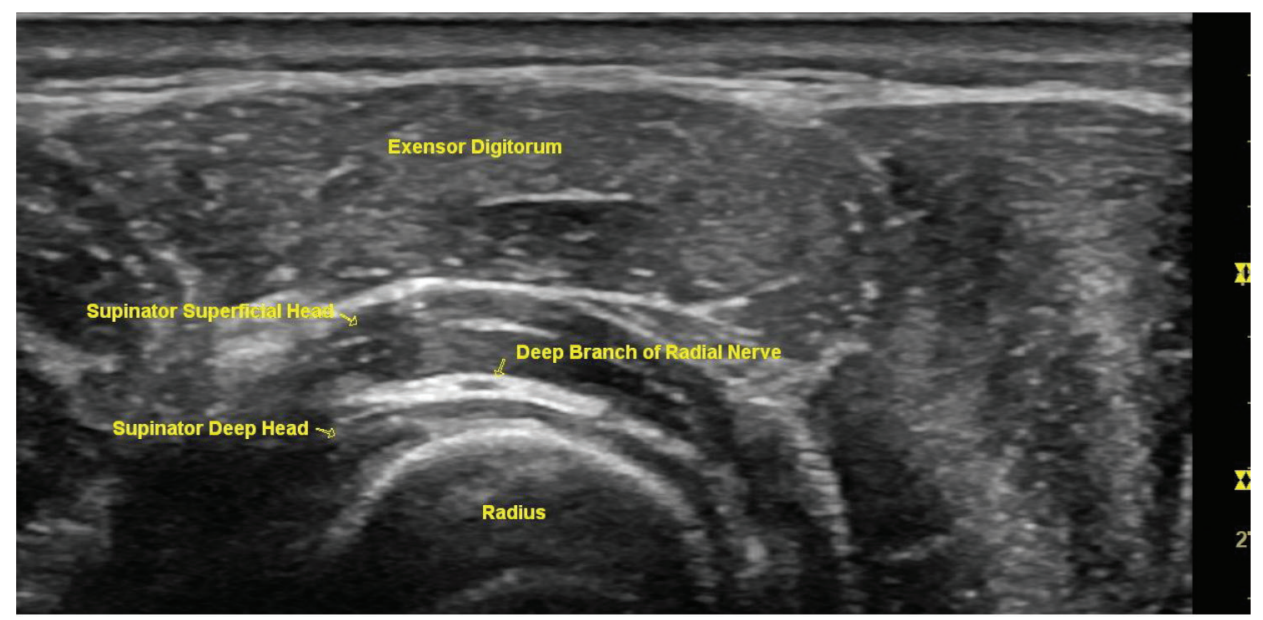

(H)
FIGURE 4-10 (Continued)

(G) The tibial nerve at the

characteristic landmarks in the tarsal tunnel. (H) The deep branch of the radial nerve at the radial tunnel identified by the superficial and deep heads of the supinator. difficult to distinguish reliably from surrounding tissue. Placing the focal zone precisely at the level of the nerve and adjusting the gray-scale mapping to optimize the contrast enhancement between the nerve and surrounding tissue will improve precision of cross-sectional area measurement. It is also helpful to scan back and forth over the area of interest to more readily appreciate the tissue contrast (Créteur et al.,

(1) 2004; VIDEO 4-6).

Measurement can be done indirectly with the use of calipers; however, most ultrasound instruments have the capability of performing direct manual tracing and automated calculation. Manual tracing is a preferable technique to placing an overlying ellipse because many nerves have an irregular circumference, particularly at sites of entrapment (FIGURE 4-17).

\section{ABNORMAL NERVE}

The imaging characteristic of abnormal nerve is often related to the nature of the injury. Focal nerve entrapments typically present with swelling proximal to the entrapment site with notching at the level of compression (FIGURES 4-18 and 4-19). There is often a loss of the fascicular architecture in long-axis view and destruction of the characteristic honeycomb pattern in short-axis view with substantial injury (FIGURES 4-20 and 4-21). In tension neuropathy, the imaging abnormalities can be relatively inconspicuous. There is a multitude of theories regarding the cause of the nerve swelling in focal neuropathy. This includes blocking of axoplasmic flow, endoneural edema, distal axonal degeneration, perineural and endoneural inflammation, perineural fibrosis, axon sprouting, remyelination, and thickening of the perineurium and epineurium (Rempel et al., 1999).

The relative echogenicity of the nerve structure tends to decrease in pathologic states (Stuart et al., 2004). This parameter is difficult to quantify precisely, but use of proximal and distal scanning as well as side-to-side comparisons can help with this assessment (FIGURE 4-22). A contributing factor to this is decreased contrast with the surrounding 


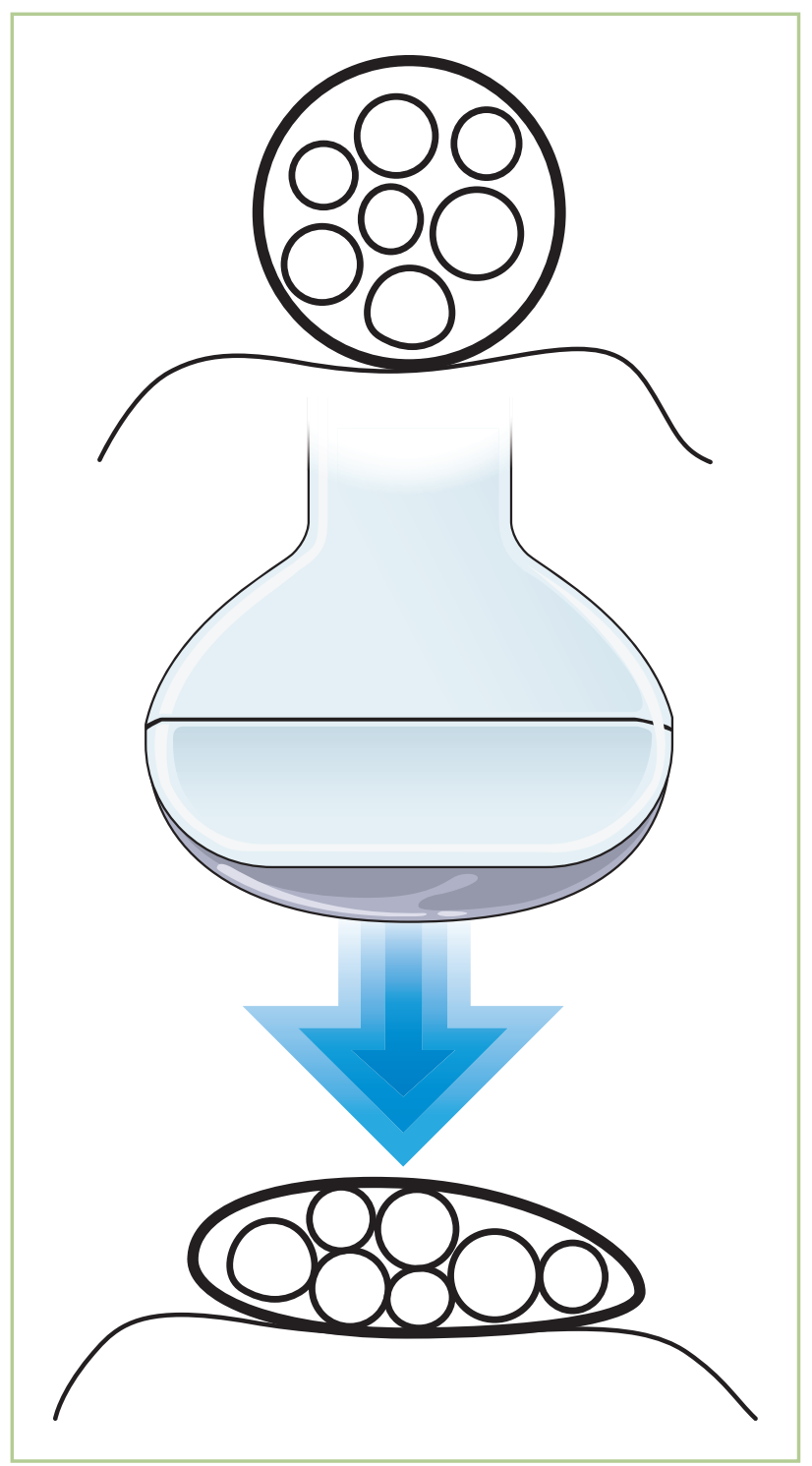

FIGURE 4-11

The effect of pressure from the transducer on the shape of a peripheral nerve. Note that the rounded shape of the epineurium seen in short axis on the top tracing is significantly flattened in the bottom tracing by pressure from the transducer. Note also that despite the significant change in the shape of the epineurium, the fascicle sizes are relatively unchanged by this external pressure.

muscle when it is denervated, or in other conditions such as myopathy. The diseased muscle echotexture becomes more isoechoic with that of peripheral nerves, resulting in less contrast.

Intraneural vascularity is also an area of developing interest. Nerves have a rich investment of endoneurial and epineurial vessels that have slow velocity. This can be recognized only rarely in some nerves with power or color Doppler (Wilder-Smith et al., 2012). Usually, no internal blood flow is seen with color Doppler in normal nerves, and therefore detectable flow signals should generally be considered to represent abnormal hypervascular change (Bianchi, 2008). Color Doppler can detect increased endoneural flow in some entrapment neuropathies (Ghasemi-Esfe et al., 2011; VIDEOS 4-7 and 4-8). Studies have shown this in both median mononeuropathies at the wrist as well as polyneuropathies (Akcar et al., 2010). The appropriate use of Doppler ultrasound for minimizing false positive and false negative assessments is discussed in further detail in Chapter 3. Caution should be used to avoid misinterpreting normal vascular flow of neighboring vessels as abnormal increased vascularity (FIGURE 4-23).

Traditionally, ultrasound has not been shown to be a reliable indicator of the relative severity of a focal neuropathy (Moran et al., 2009); however, advancements with assessment of peripheral nerve and muscle echotexture have led to improvements in this area. The classifications of Seddon and Sunderland (TABLE 2-1) are frequently used when assigning the degree of nerve injury severity. Precise delineation of these categories is not always possible sonographically, but some distinct patterns are seen, depending on the nature of the neuropathy. Diffuse cross-sectional area enlargement is most consistently seen with demyelination in generalized neuropathies (Grimm et al., 2014). Focal neurapraxic injuries often display localized enlargement with fairly preserved fascicular pattern. There is significant variation seen with peripheral nerves displaying conduction block. There can be focal swelling of the whole nerve at the site of the injury, enlargement of a single fascicle, or even a relatively indistinct difference from the majority of the length of the nerve in a more diffuse abnormality (FIGURE 4-24).

In axonotmesis there can also be diffuse swelling, but there is often a loss of the fascicular pattern. Other features frequently seen in axonotmesis include an irregular nerve caliper as well as areas of interneural fluid or even a homogeneously hypoechoic appearance. By contrast, an "empty bed" appearance with the retracted nerve endings occur in neurotmesis. Although it is not always easy to reliably distinguish between axonotmesis and neurotmesis on ultrasound, this remains an important role for imaging because this distinction cannot be made with electrodiagnosis before reinnervation (Tagliafico et al., 2010). Recent improvements with ultra-high-frequency transducers are providing 


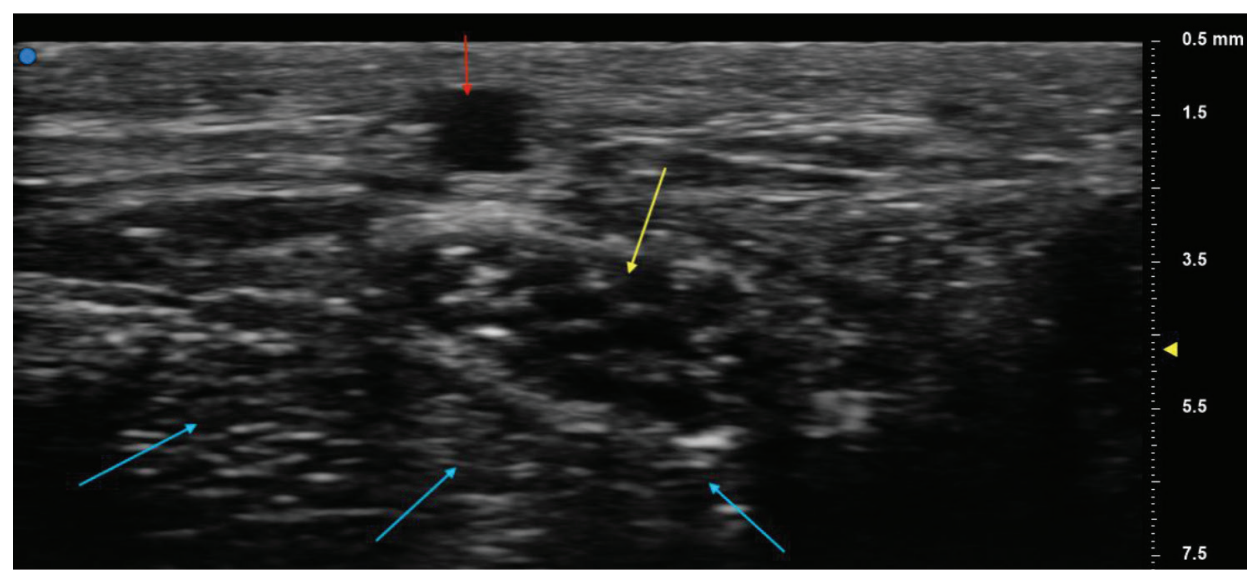

(A)

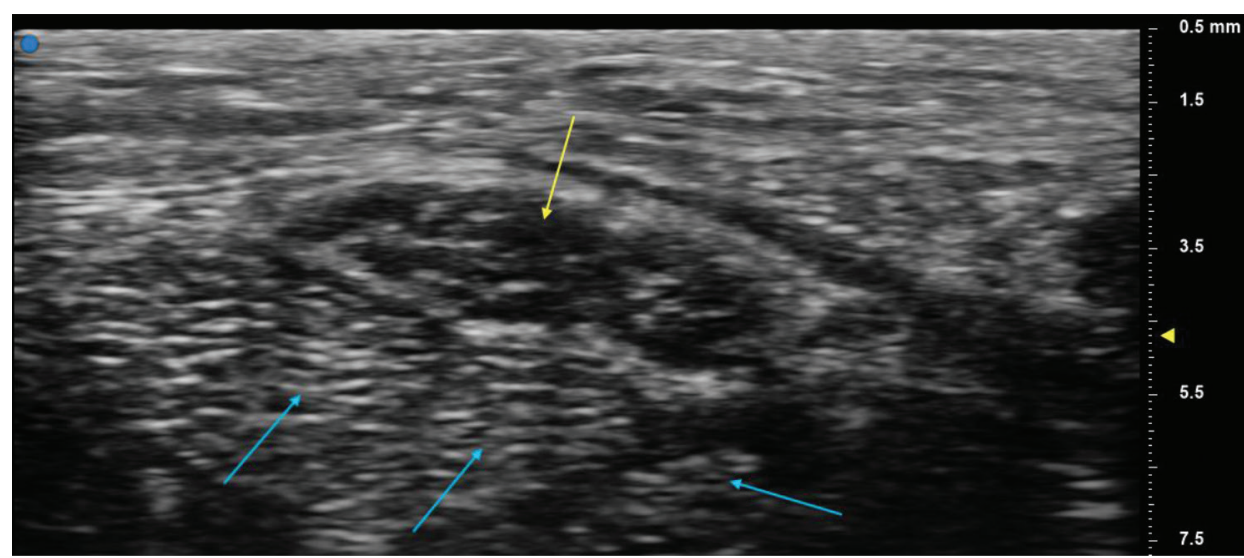

(B)

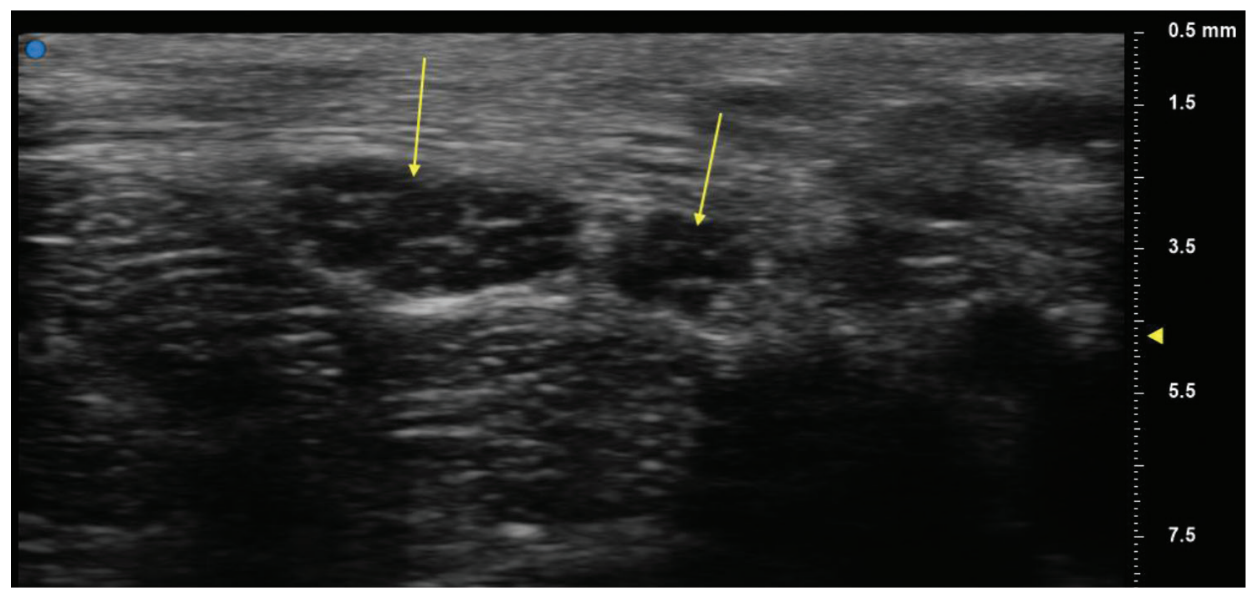

FIGURE 4-12

Sonograms demonstrating the effect of pressure from the transducer on peripheral nerves. (A) There is very light transducer pressure on the median nerve (yellow arrow) at the proximal carpal tunnel. Note also the surrounding tissue including a superficial vein (red arrow) and the neighboring flexor digitorum superficialis tendons (blue arrows). (B) With heavier transducer pressure, there is relative flattening of the nerve (yellow arrow). Note also that the overlying vein disappears with the transducer pressure and the surrounding tendons become somewhat more conspicuous.
FIGURE 4-13

Sonogram of a bifid median nerve (yellow arrows) at the carpal tunnel inlet. greater conspicuity of fascicular architecture, potentially leading to improved distinction of injury patterns (Cartwright et al., 2017).

Measurement of nerve diameter, as well as assessment of nerve echotexture, should also be correlated in longitudinal plane when assessing for pathologic swelling and fascicular disruption (FIGURE 4-25). Similar to the imaging of tendons and muscles, abnormalities should always be identified in at least two planes for confirmation. For peripheral nerve measurement, the long-axis plane is more challenging to maintain in full view and often requires 
FIGURE 4-14

Sonograms demonstrating the effect of alteration of frequency on the appearance of a longitudinal image of the median nerve (yellow arrows) at the carpal tunnel. Panel ( $A$ ) is at a higher frequency of $15 \mathrm{MHz}$. Note the relative clarity of the more superficial proximal portion of the nerve (left side of the image) and the relative loss of resolution more distally as the nerve traverses under the thicker flexor retinaculum at the carpal tunnel outlet (right side of the image). Panel (B) is at $8 \mathrm{MHz}$. Note that at the lower frequency, the detailed architecture of the more superficial portion of the median nerve is less evident but the more distal portion is easier to identify. Also note the improved resolution of the underlying flexor tendons and other tissue. These images demonstrate the value of assessing the region of interest around the peripheral nerve at more than one frequency.

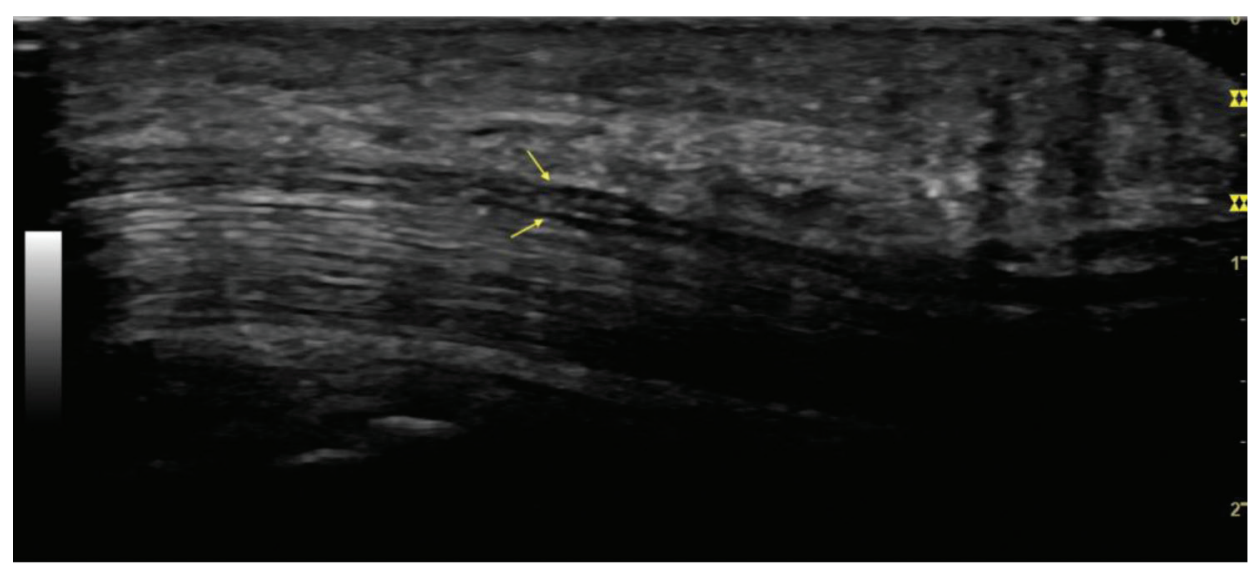

(A)

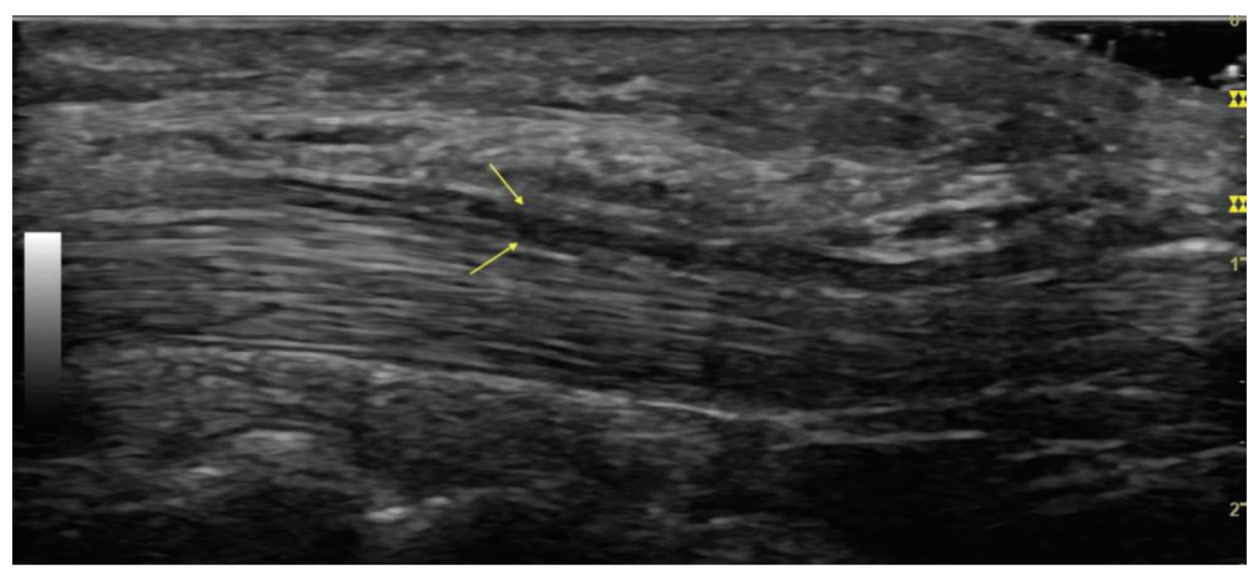

(B)

\section{FIGURE 4-15}

Sonogram of the sciatic nerve (yellow arrow) at mid-thigh. Note the hypoechoic nerve fascicles (red arrow) within the hyperechoic epineurium (blue arrow).

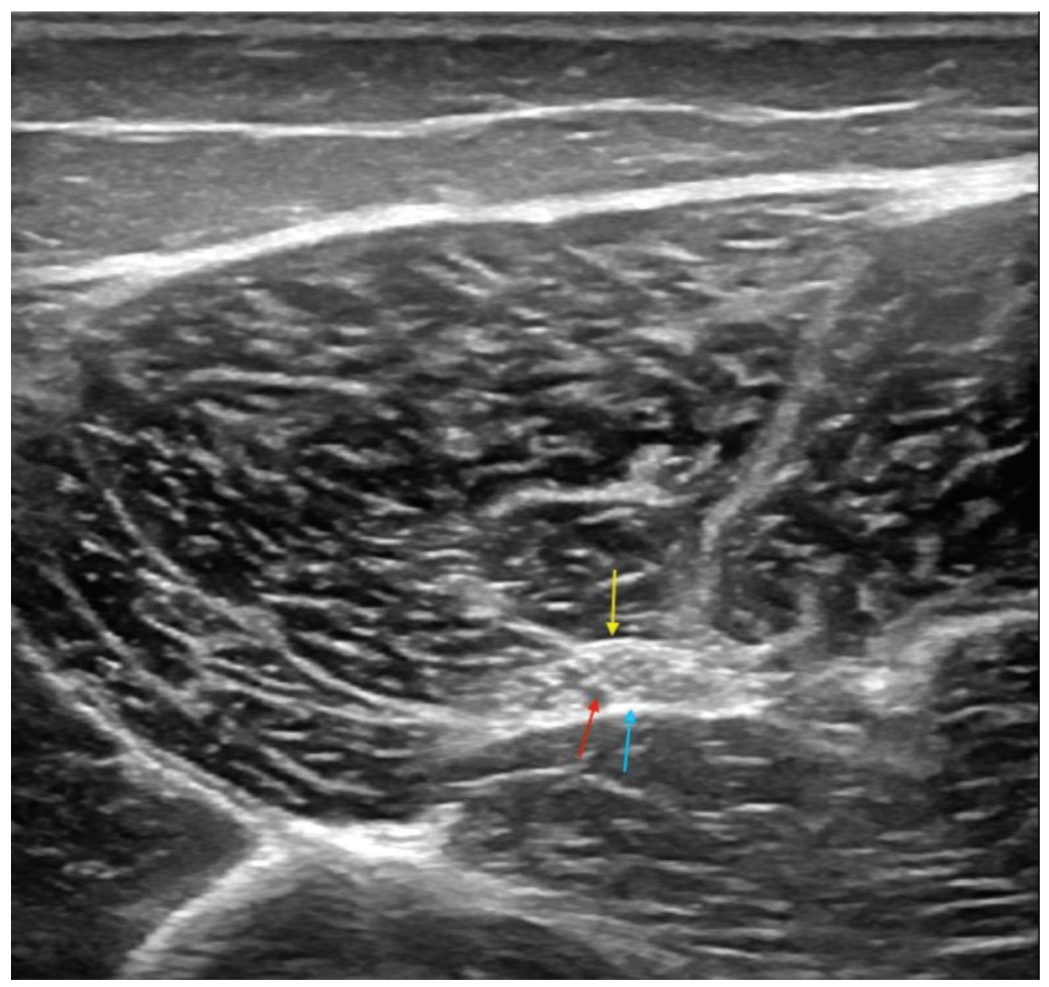




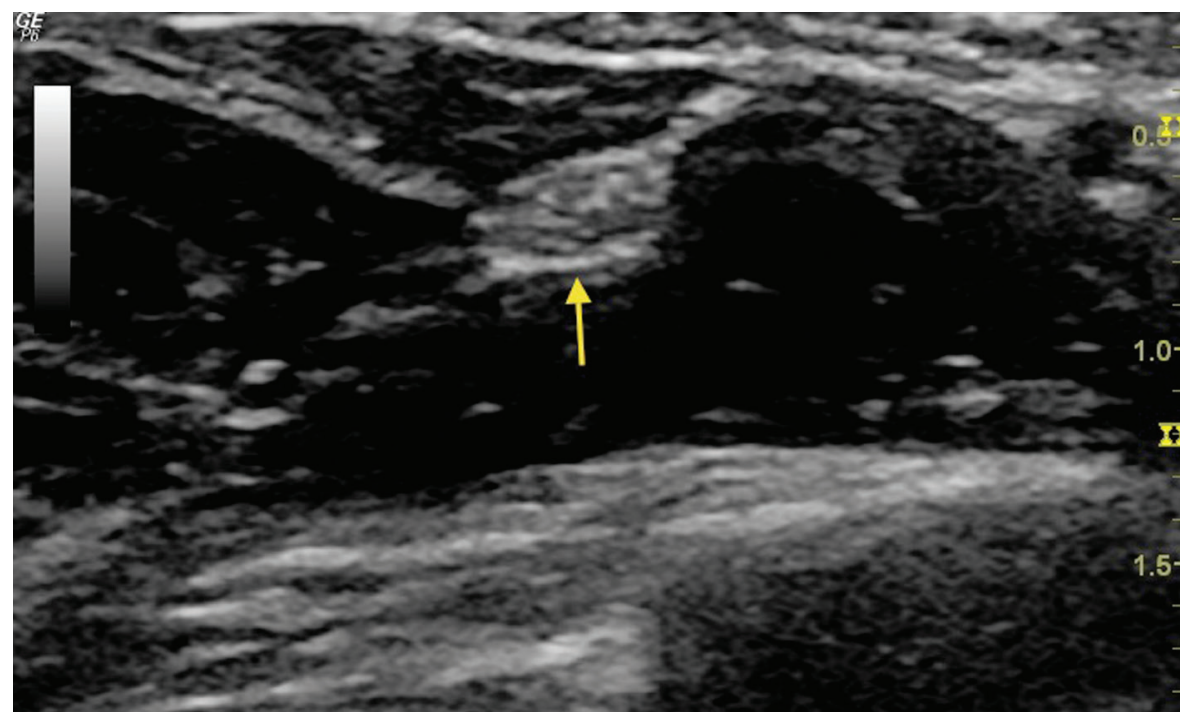

(A)

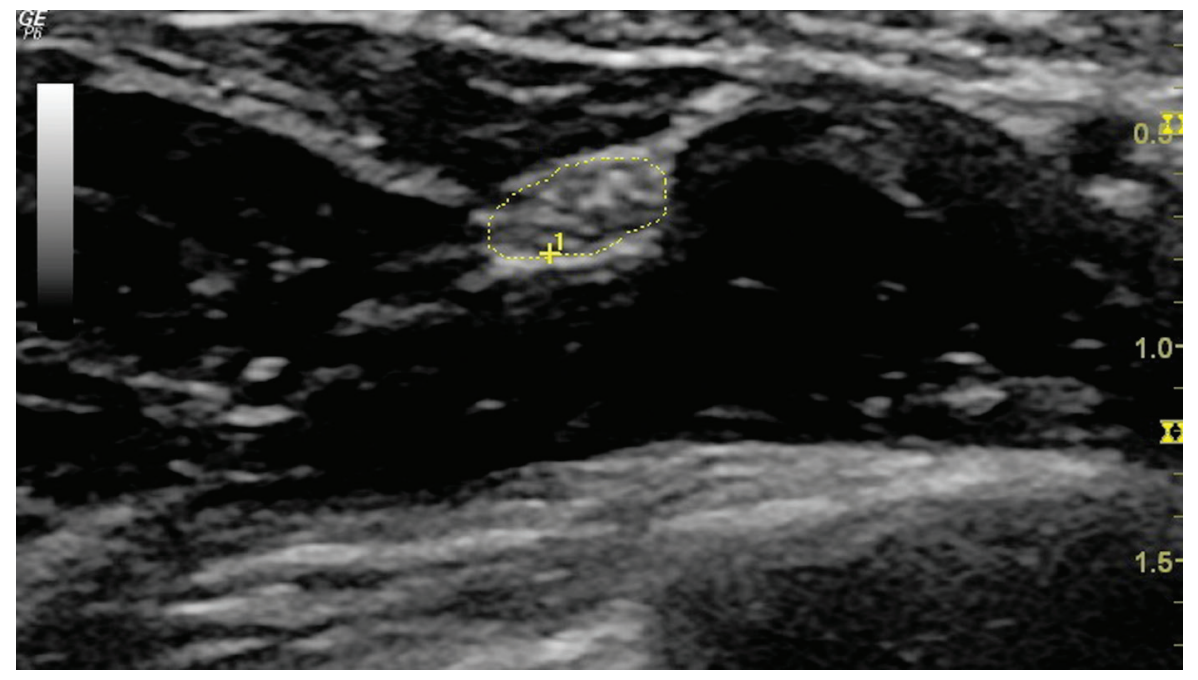

(B)

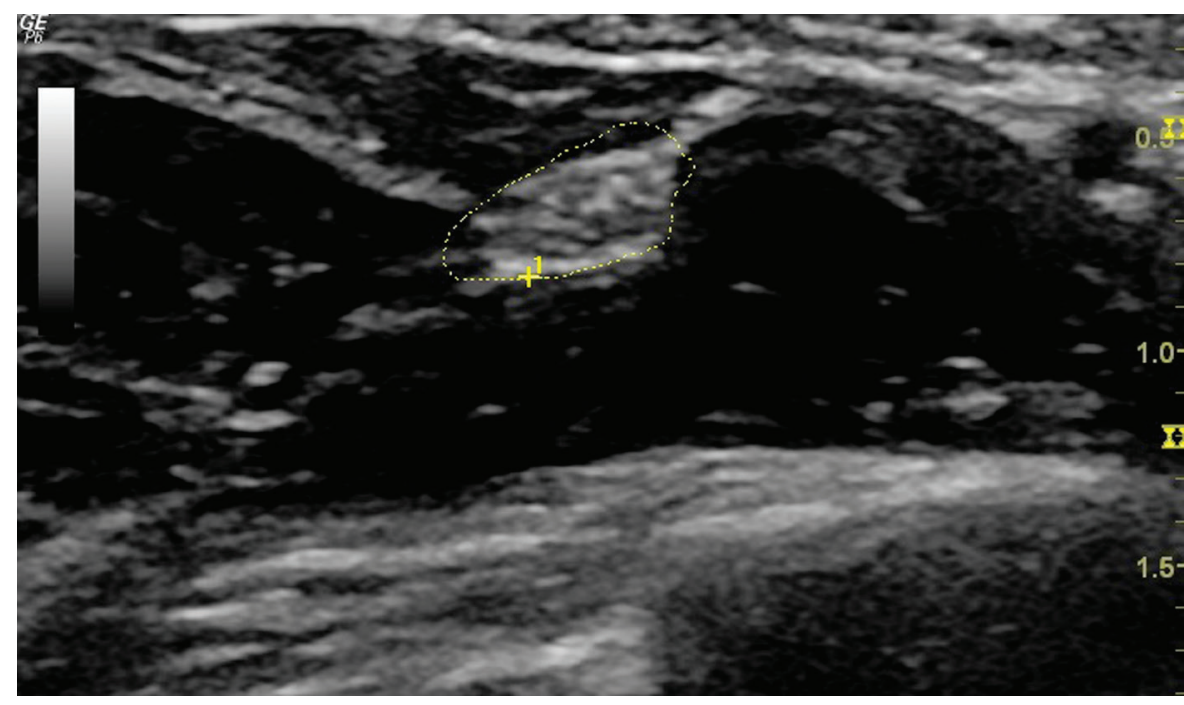

(C)
FIGURE 4-16

Sonograms of proper direct measurement technique of the cross-sectional area. (A) The nerve (yellow arrow) is seen in short axis. The hypoechoic nerve fascicles and hyperechoic epineurium are evident. (B) The cross-sectional area is properly measured by tracing between the hypoechoic fascicles and just inside the hyperechoic outer epineurium. (C) The measurement is performed improperly with tracing in and outside the outer epineurium. This results in an inconsistent and incorrectly increased cross-sectional area calculation. 


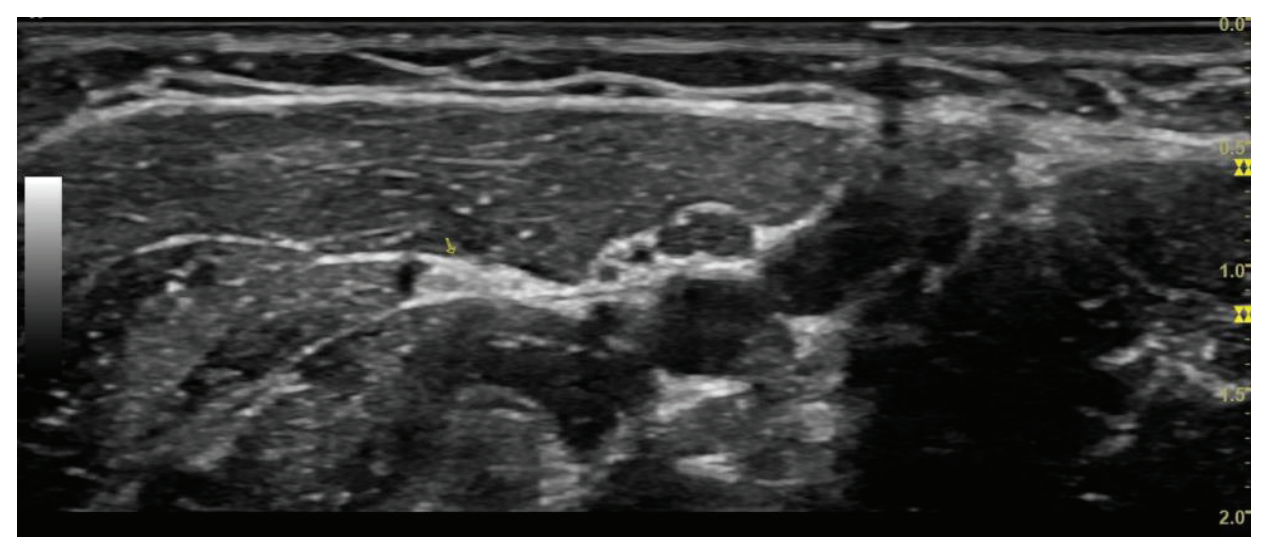

(A)

\section{FIGURE 4-17}

Sonograms demonstrating the varying shapes of peripheral nerves in short axis, making it difficult to use a simple ellipse measurement for a reliable cross-sectional area. All nerves are depicted by a yellow arrow: (A) musculocutaneous nerve in the arm, (B) radial nerve above the elbow, (C) ulnar nerve at the medial epicondyle.

(continued)

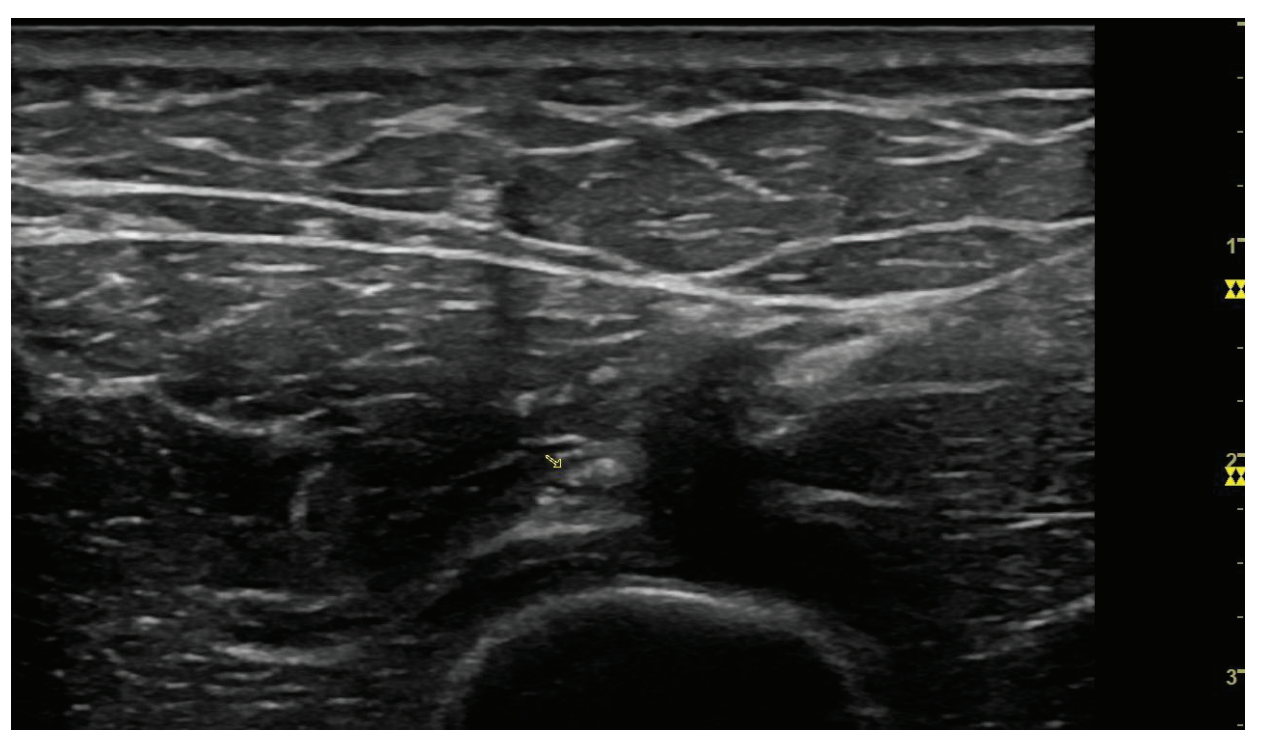

(B)

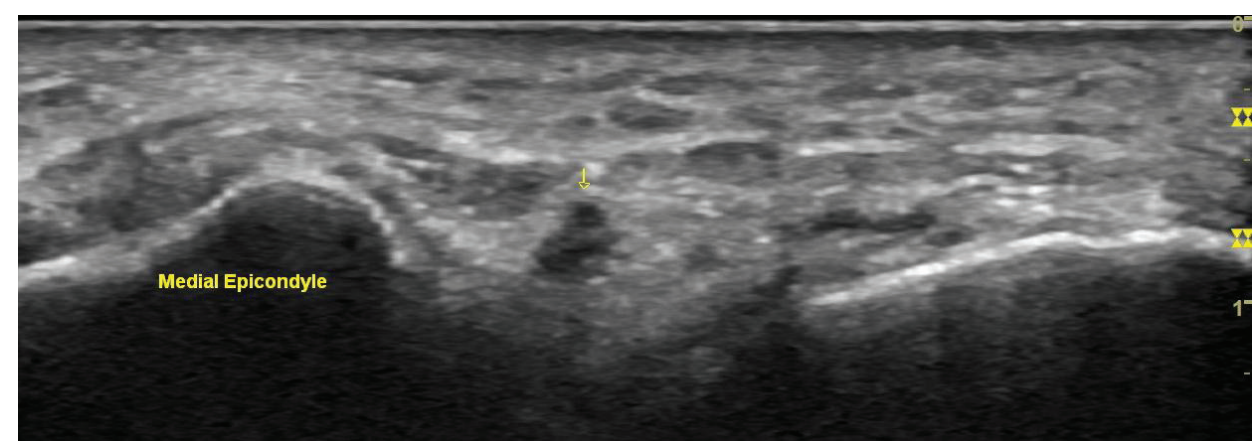

(C) multiple measurements with movement of the transducer along its course. Short-axis, cross-sectional areas can be erroneously calculated if the nerve is oblique to the transducer or if the angle of incidence from the transducer is not orthogonal to the nerve. Good correlation between the short-axis and longaxis views should be confirmed to establish accuracy of measurement. This will also alleviate concern for error based on inappropriate positioning of an image (FIGURE 4-26).

\section{Nerve Trauma}

Nerves tend to develop bulbous swelling at the level of a transection or other significant injury 

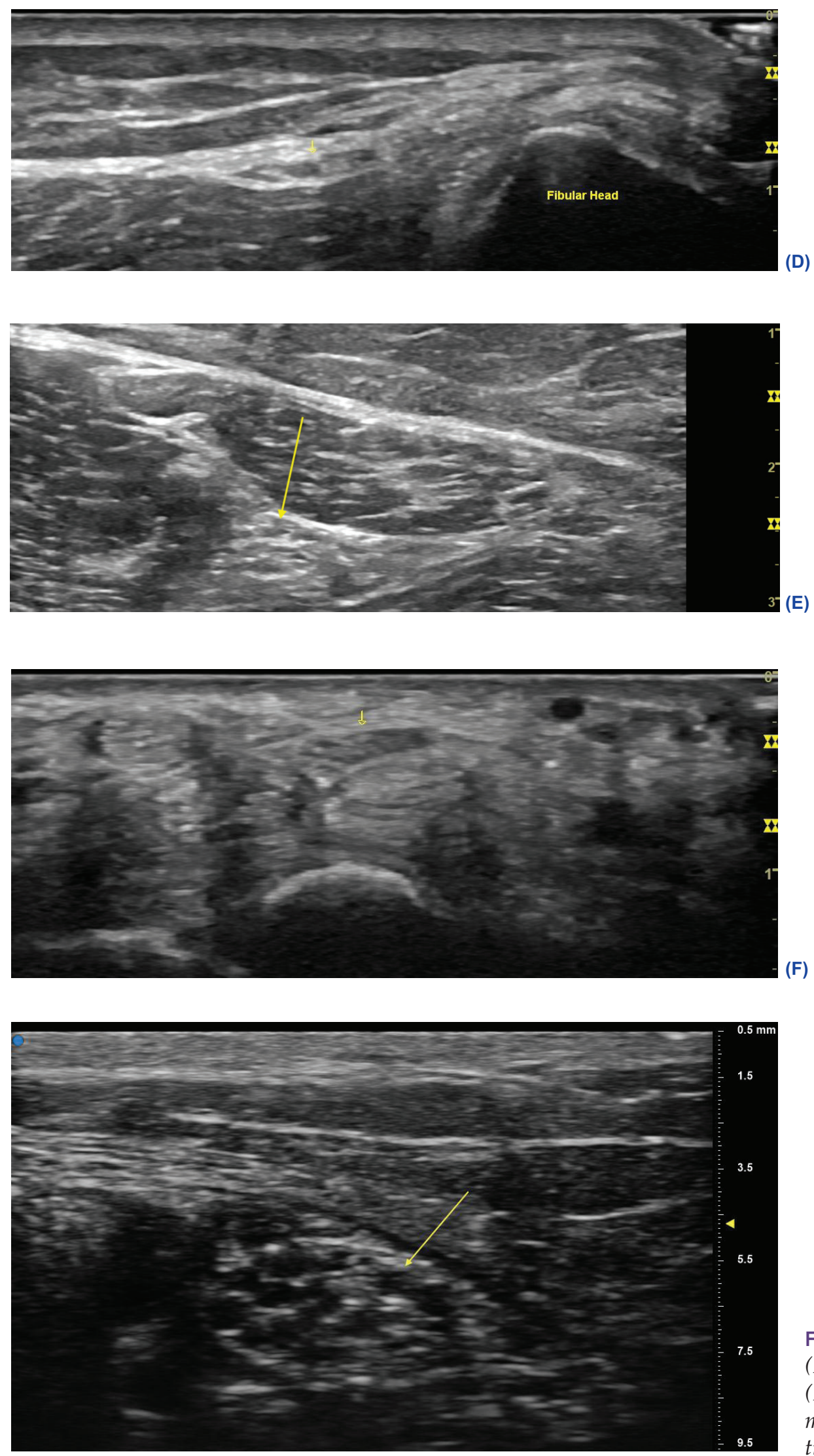

FIGURE 4-17 (Continued)

(D) fibular nerve at the fibular head, (E) sciatic nerve in the thigh, $(F)$ median nerve at the wrist, and $(G)$ (G) tibial nerve at the tarsal tunnel. 
(FIGURE 4-27). Ultrasound has good reliability for finding transected nerves (Cartwright, Chloros et al., 2007) and focal neuromas. Traumatic neuromas can sometimes be localized by palpation; however, not all neuromas create palpable tenderness, and other traumatized tissue can be the source of localized discomfort. A neuroma is confirmed with ultrasound by identifying the well-defined hypoechoic mass along the path of the injured nerve (Fornage, 1988; FIGURE 4-28). Back-and-forth scanning along the path of the injury can improve the conspicuity of

- neuromas in even relatively small nerves (VIDEO 4-9). Scanning in posttraumatic conditions should also include a detailed assessment of the surrounding tissue for potential effect on the nerve. Identification of potentially confounding factors such as compressive bone fragments, vascular clots or injuries, unstable joint movement, hematomas, infections, and scar tissue can assist with appropriate diagnosis and management.

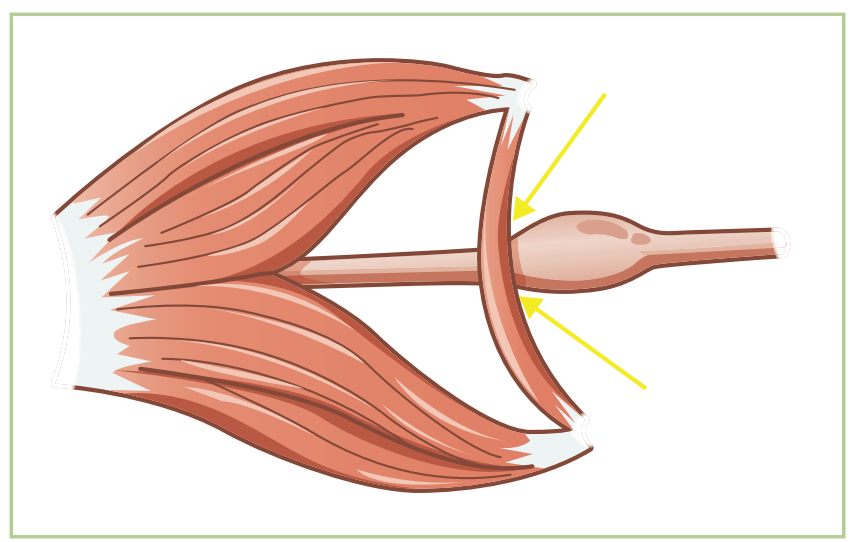

FIGURE 4-18

A focal nerve entrapment with swelling proximal to the compression sites and notching (yellow arrows) at the entrapment site.

\section{FIGURE 4-19}

Sonograms of a notch sign (yellow arrowheads) of the $(A)$ ulnar nerve at the elbow and $(B)$ median nerve at the carpal tunnel. Note the focal enlargement just proximal to the area of narrowing.

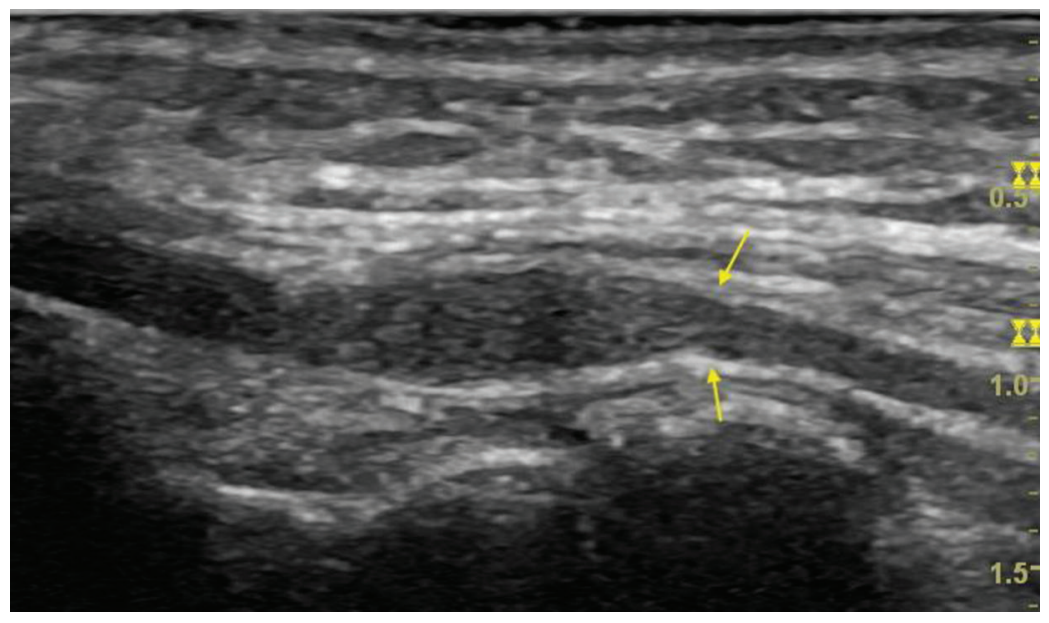

(A)

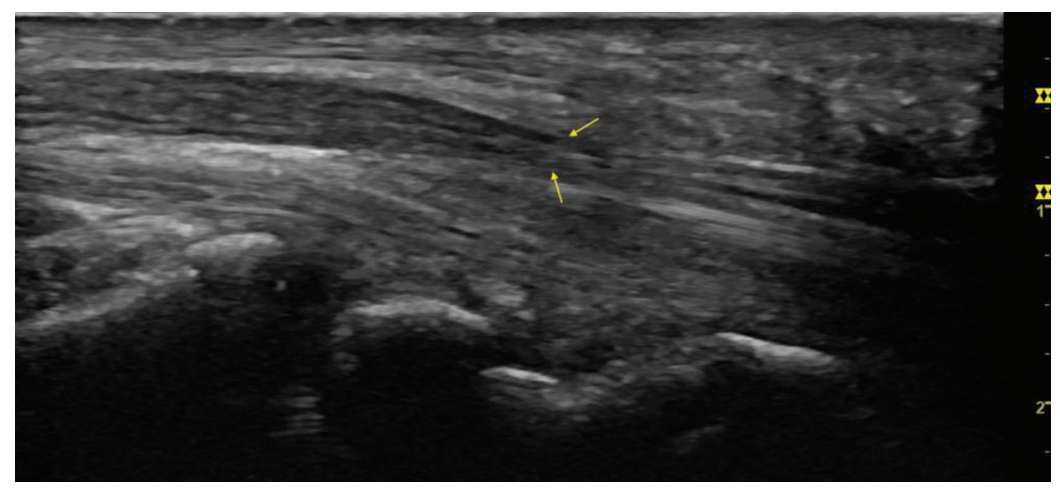

(B) 


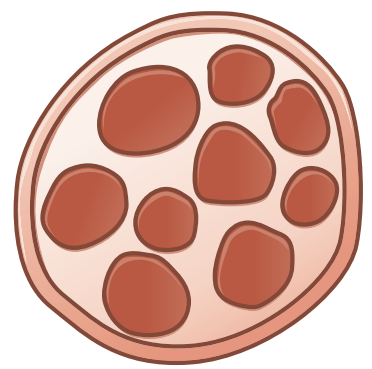

(A)

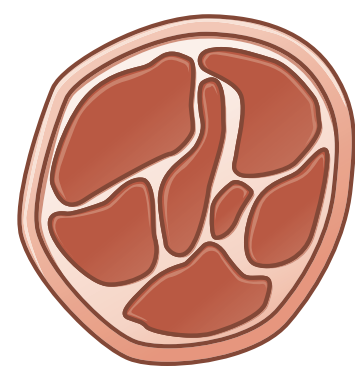

(B)

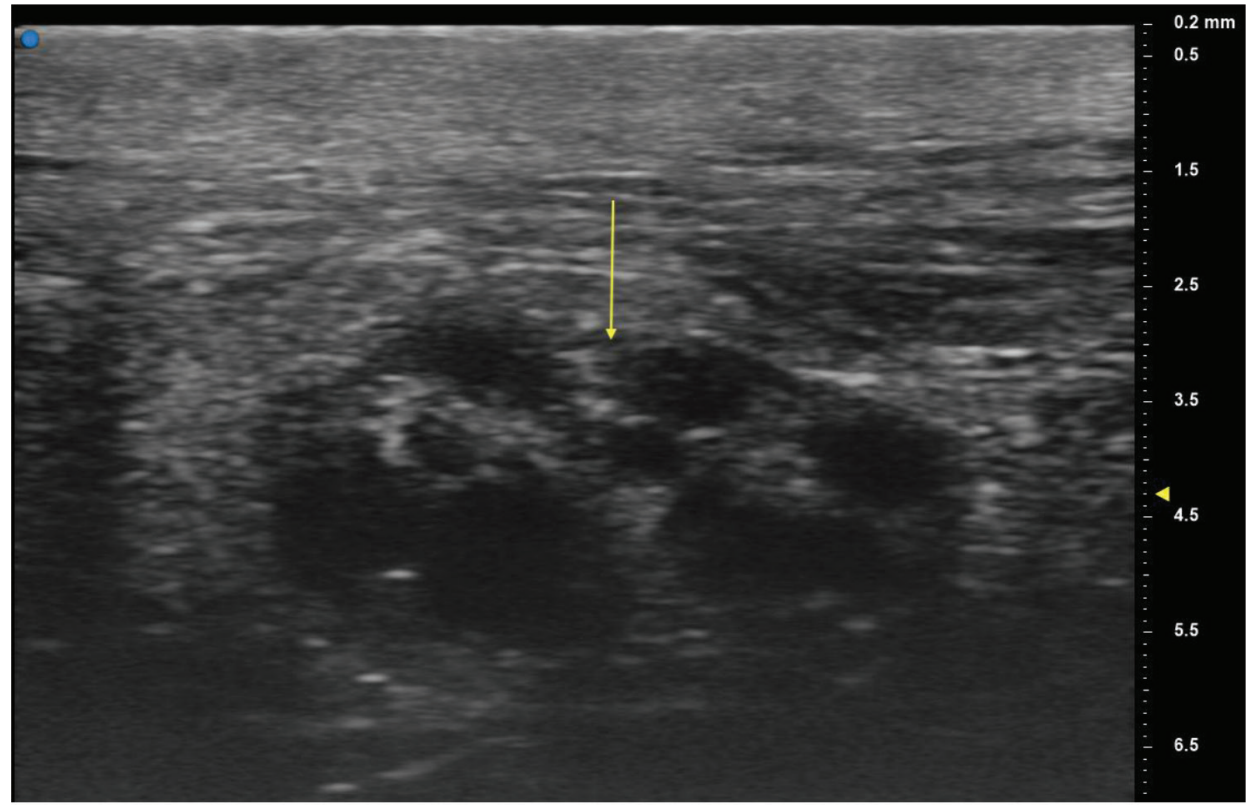

(C)
FIGURE 4-20

Disruption of the fascicular architecture of a peripheral nerve seen in short-axis view. (A) Illustration of the normal fascicular architecture pattern. (B) The fascicles are enlarged and irregular denoting neuropathy. (C) Sonogram of a common fibular neuropathy with markedly enlarged fascicles.

\section{Use in Generalized Peripheral Neuropathies}

Nerve changes are also seen in some generalized peripheral neuropathies. There is not consistent correlation with nerve swelling and clinical and electrodiagnosic abnormalities in all generalized neuropathies. Ultrasonography has been studied in hereditary and acquired peripheral neuropathies, leprosy, and some other conditions. The examiner should assess for both focal and fusiform nerve swelling. Ultrasound can also be helpful in identifying focal neuropathies within the context of underlying generalized peripheral neuropathy. Approaches to generalized peripheral neuropathies are discussed in more detail in Chapter 16.

\section{INTERFACE WITH ELECTROPHYSIOLOGIC TESTING}

Ultrasound is a valuable tool for overcoming challenges in an electrodiagnostic laboratory. Ultrasound can assist with confirming localization of needle electrode placement in challenging muscles for both improved accuracy and avoidance of adjacent vital structures. This is particularly useful with inexperienced examiners (Boon et al., 2008, 2011). The anatomic clarity and dynamic visualization seen with ultrasound of muscle and nerve can provide invaluable learning experience for physicians in training and offers "hands-on" exposure previously only obtained with cadavers. Ultrasound provides localization of peripheral nerves, for both 


\section{FIGURE 4-21}

Sonograms of a severe ulnar neuropathy at the elbow displaying focal disruption of the fascicular architecture. (A) The shortaxis view of the nerve (yellow arrow) at the cubital tunnel displays enlargement of the fascicles. (B) The short-axis view of the ulnar nerve (yellow arrow) more proximally in the ulnar groove displays a loss of the normal detail of the fascicular architecture. (C) The longaxis view of the nerve corroborates the hypoechoic appearance of the fascicular injury (yellow arrows). Note also, the hyperechoic appearance of the flexor carpi ulnaris (FCU 1) and FCU 2 in (A) and blue arrow in $(C)$. This is consistent with denervation related to relative severity of the ulnar neuropathy.

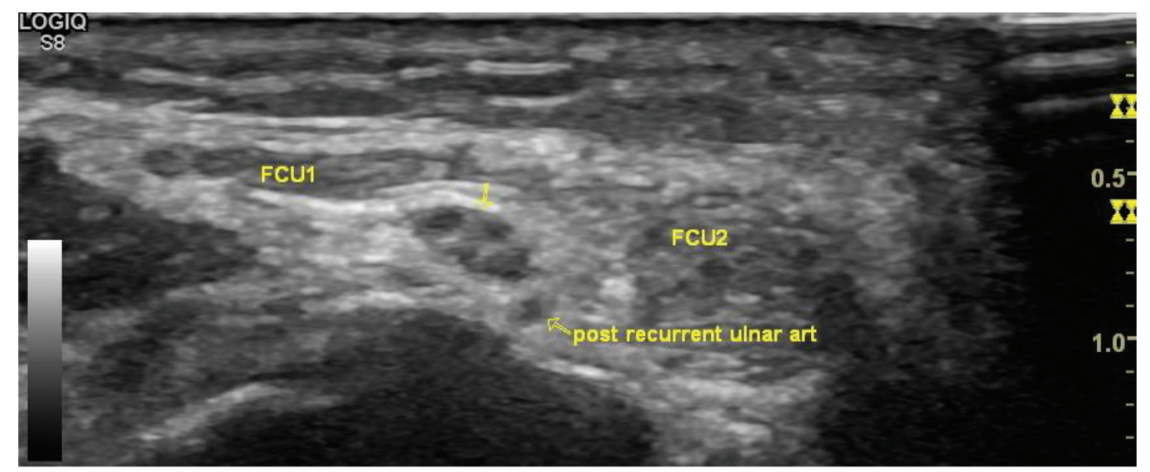

(A)

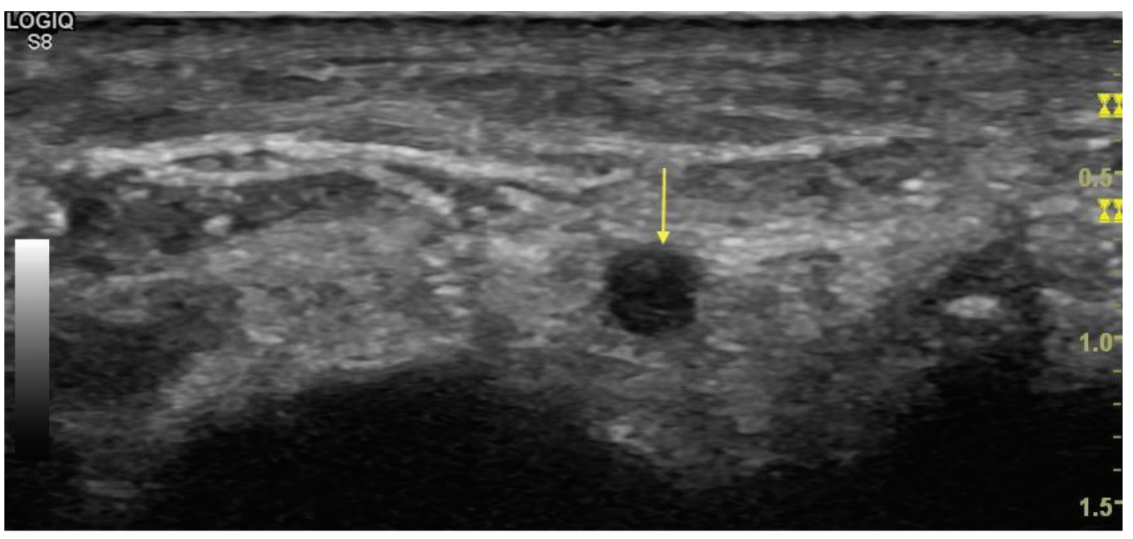

(B)

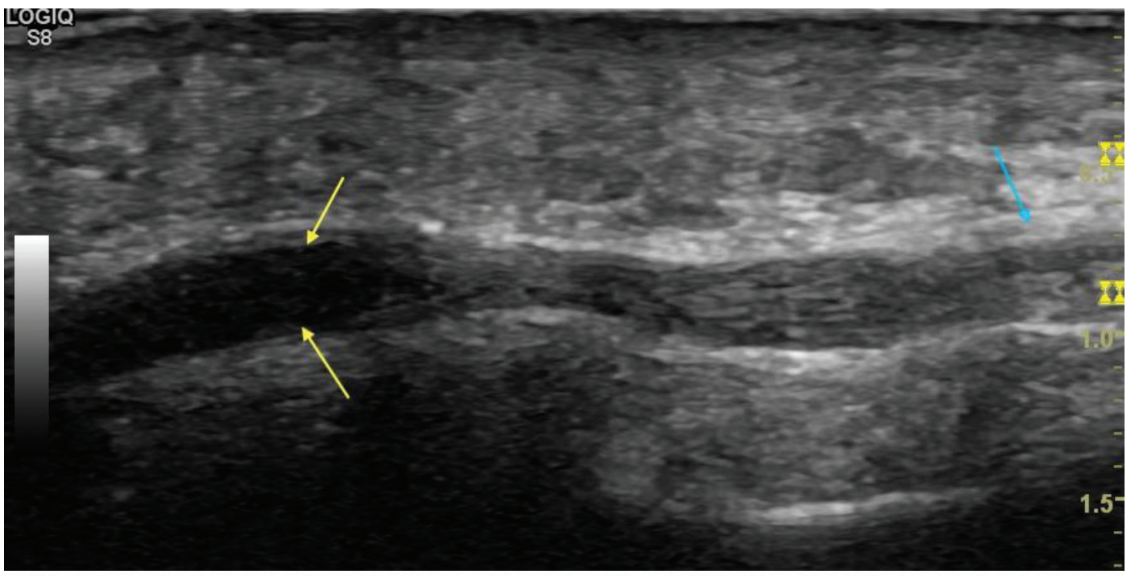

(C) stimulation and recording, in better detail than surface anatomy alone. Ultrasound guidance is typically not needed by experienced practitioners to perform routine nerve conduction studies. It can be helpful for challenging or small nerves that are less commonly studied. It can also be helpful for electrode placement when performing near-nerve conduction studies. Ultrasound can also be used to identify anatomic variants.

Ultrasound provides anatomic information that is complementary to the electrophysiologic findings. The diagnostic value of its routine use in conjunction with electrodiagnostic studies has been demonstrated in both peripheral nerve diseases and trauma (Gallardo et al., 2015; Padua et al., 2012; Padua et al., 2013). There is growing support for the incorporation of the regular use of ultrasound in electrodiagnostic laboratories and clinics for improving diagnostic acumen in a broad range of neuromuscular conditions (Walker et al., 2018). 

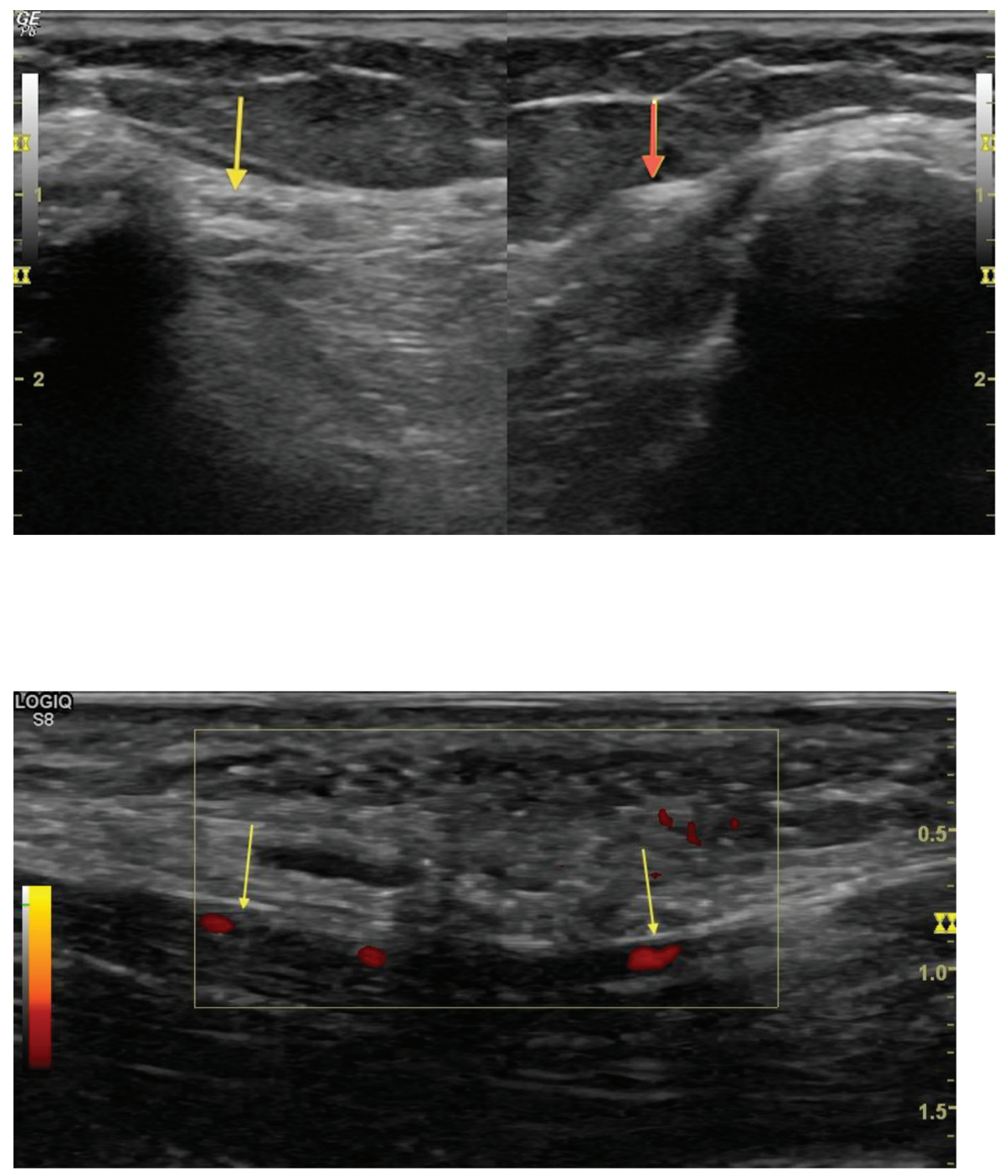

(A)

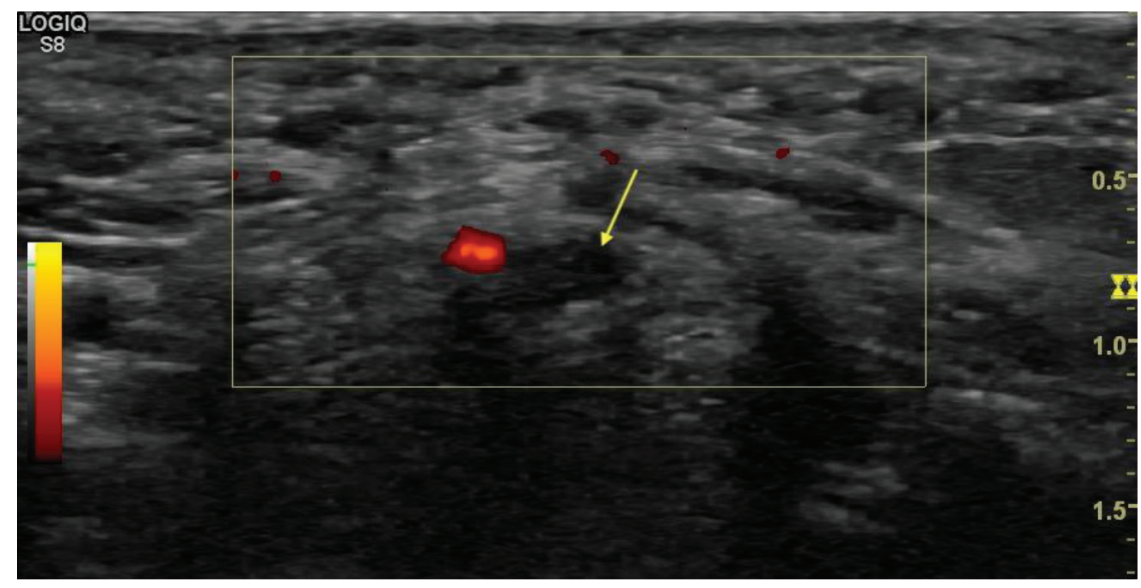

(B)

\section{FIGURE 4-22}

Sonograms of the difference in echogenicity in a pathologic state. The image on the left is of an abnormal fibular nerve (yellow arrow) that appears hypoechoic relative to the normal fibular nerve (red arrow) on the right.

\section{FIGURE 4-23}

Sonograms demonstrating a potential source of misinterpretation when assessing vascularity of a peripheral nerve. (A) Long-axis view of the median nerve (yellow arrows) with increased power Doppler flow. (B) Short-axis view of the same nerve (yellow arrow) reveals the Doppler signal is related to a neighboring persistent median artery and not increased intraneural flow. Detailed inspection should be used to avoid confusing neovascularization with flow from neighboring vessels or flash artifact. 
FIGURE 4-24

Potential findings with a nerve that displays conduction block. (A) The nerve appears to have focal swelling but relatively normal fascicular architecture. (B) The nerve does not appear swollen but there is a dramatically enlarged fascicle. (C) There is no discernable structural abnormality.
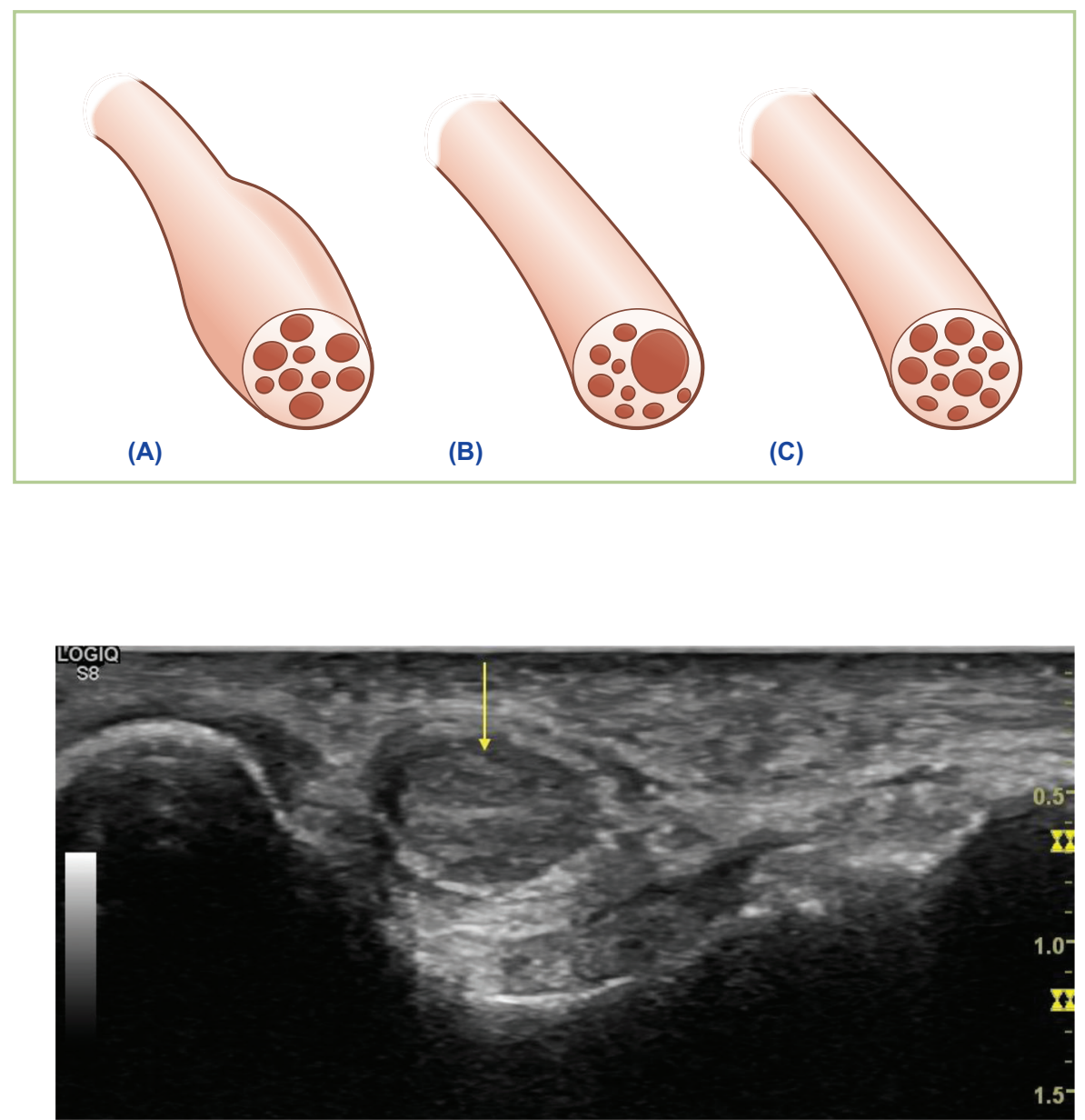

(A)

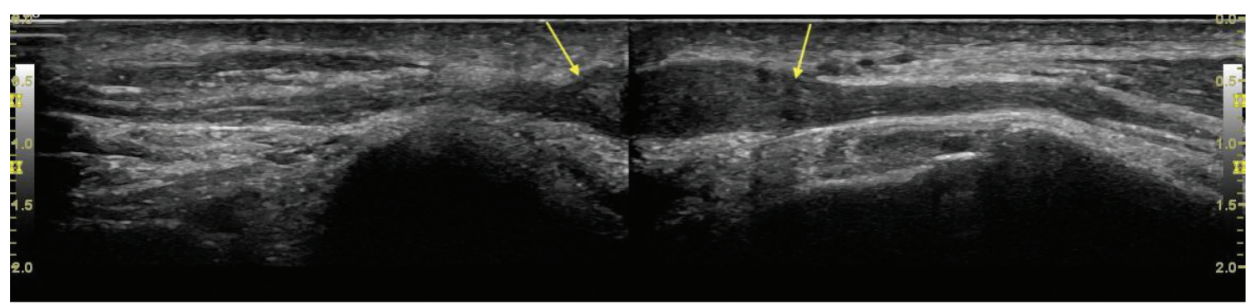

(B)

\section{TUMORS THAT CAN AFFECT PERIPHERAL NERVES}

Evaluation of tumors in and around peripheral nerves can be challenging. Knowledge of typical sites of various tumors as well as their sonographic appearances can assist in reliably characterizing some masses encountered during an ultrasound study (Sintzoff, Gillard et al., 1992). The use of a detailed history and physical examination remains a critical element of this assessment. MRI is often the favored imaging modality for evaluating tumors and masses. Despite this, the high resolution and Doppler flow capabilities of ultrasound make it a useful modality for assessing many tumors (Bendix et al., 2005). Color Doppler is particularly helpful for determining the vascularity of tumors (FIGURE 4-29, VIDEOS 4-10 and 4-11). 


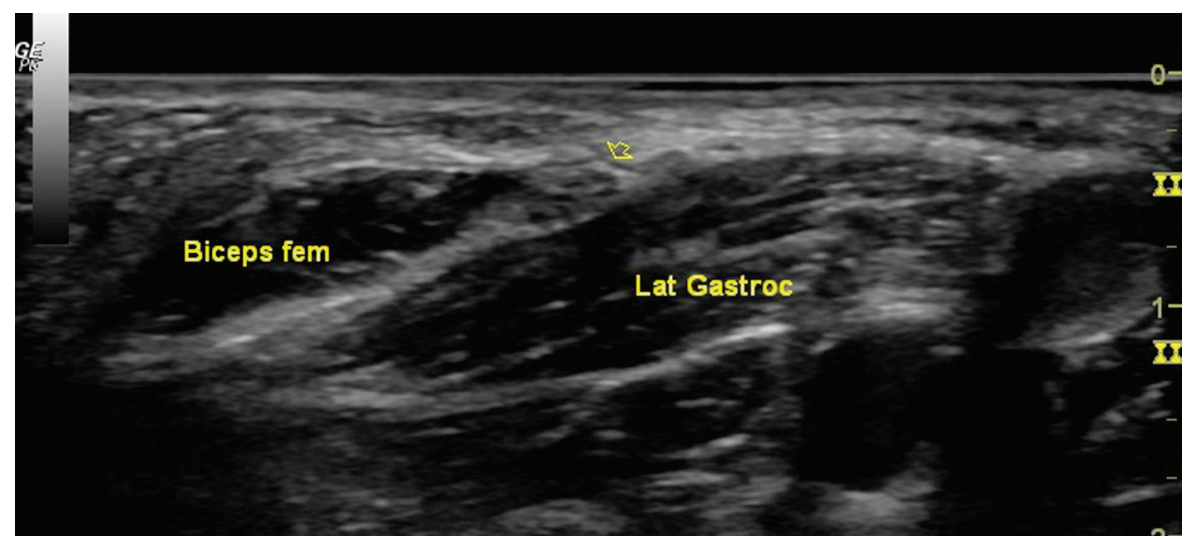

(A)

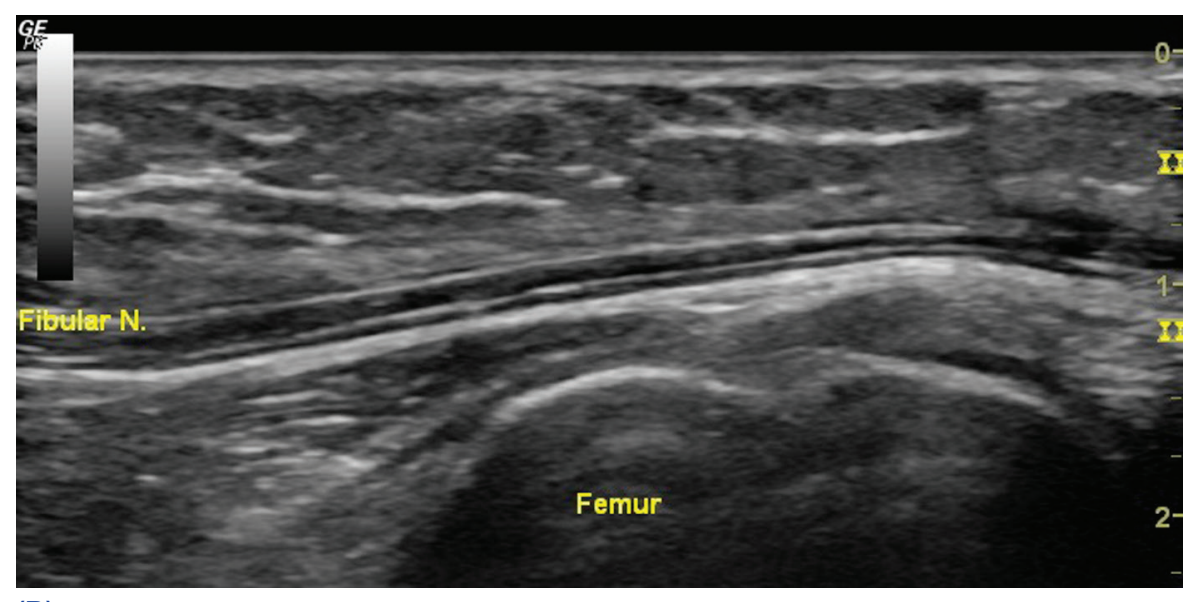

(B)

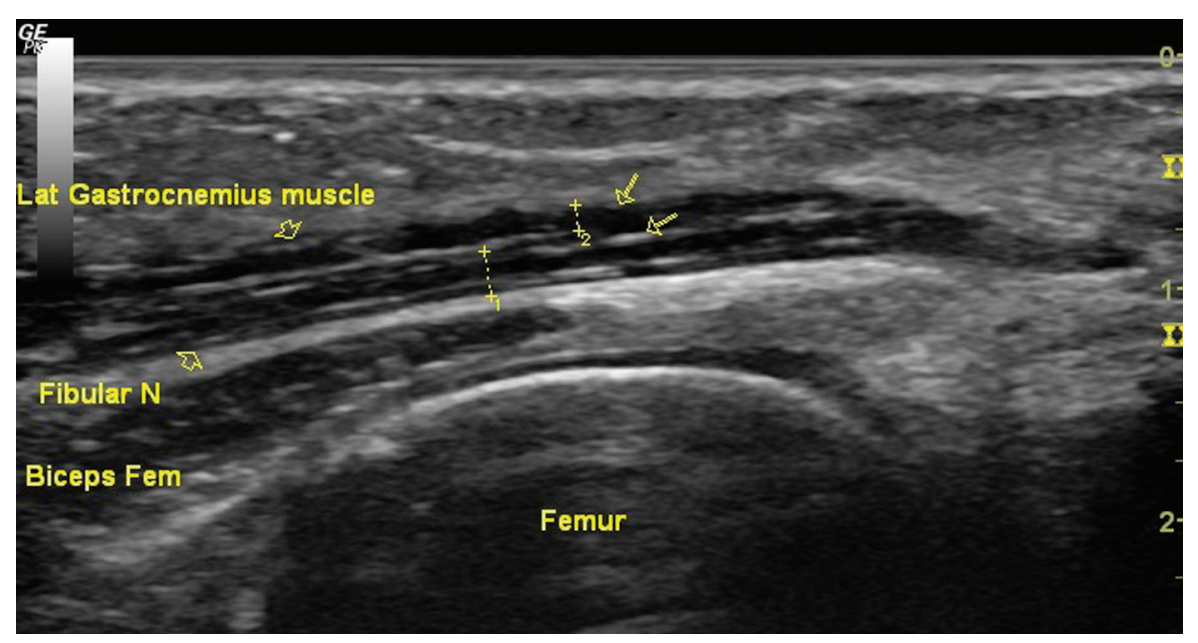

(C)

\section{FIGURE 4-26}

Demonstration of a potential source of measurement error in nerves in long axis. The image in $(A)$ demonstrates a normal common fibular nerve in short axis (yellow arrowhead). (B) The same nerve is seen with appropriate scanning in long axis. (C) The same common fibular nerve is shown with the transducer moved slightly medially. Just superior to the nerve (measurement 1), the similarly hypoechoic lateral gastrocnemius muscle appears (measurement 2). If not recognized, this could potentially be misinterpreted as an enlarged nerve. Comparing the nerve appearance in two views can prevent this error.
Indications for imaging suspected tumors include nerve entrapment at an atypical site or sites, mass lesions, and other unusual presentations of neuropathy. Imaging allows the tumor to be localized precisely as well as characterized (FIGURE 4-30). It is also important to identify the location of the nerve in relation to the mass. Ultrasound cannot always reliably differentiate types of tumors but can define 
FIGURE 4-27

The bulbous swelling associated with severe axonotmesis of a peripheral nerve.

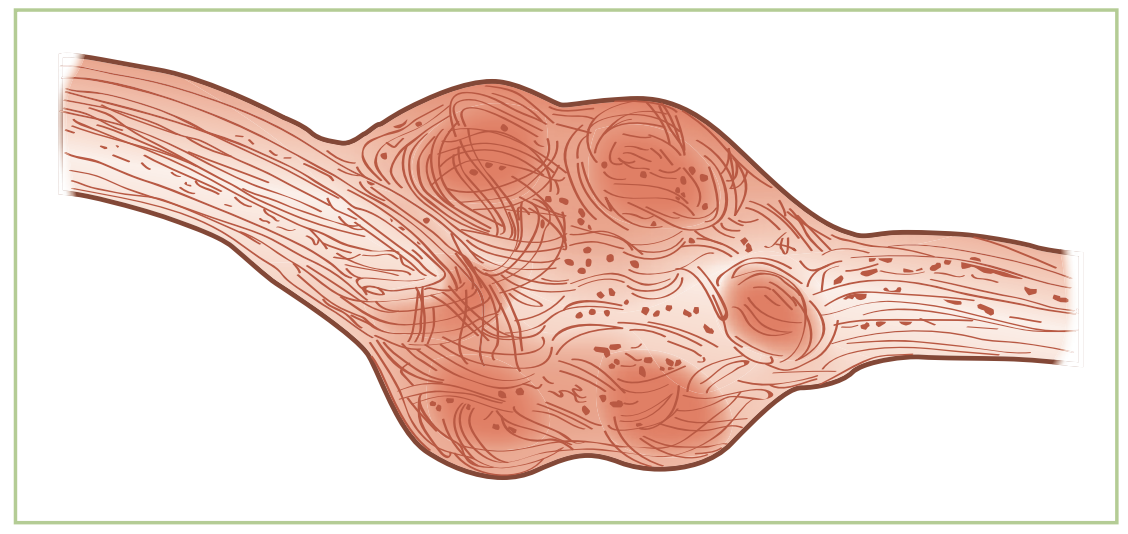

FIGURE 4-28

Sonogram of a focal nerve injury from trauma creating a neuroma. Note the focal enlargement of the nerve (shown in long axis) and disruption of the normal fascicular architecture at the injury site.
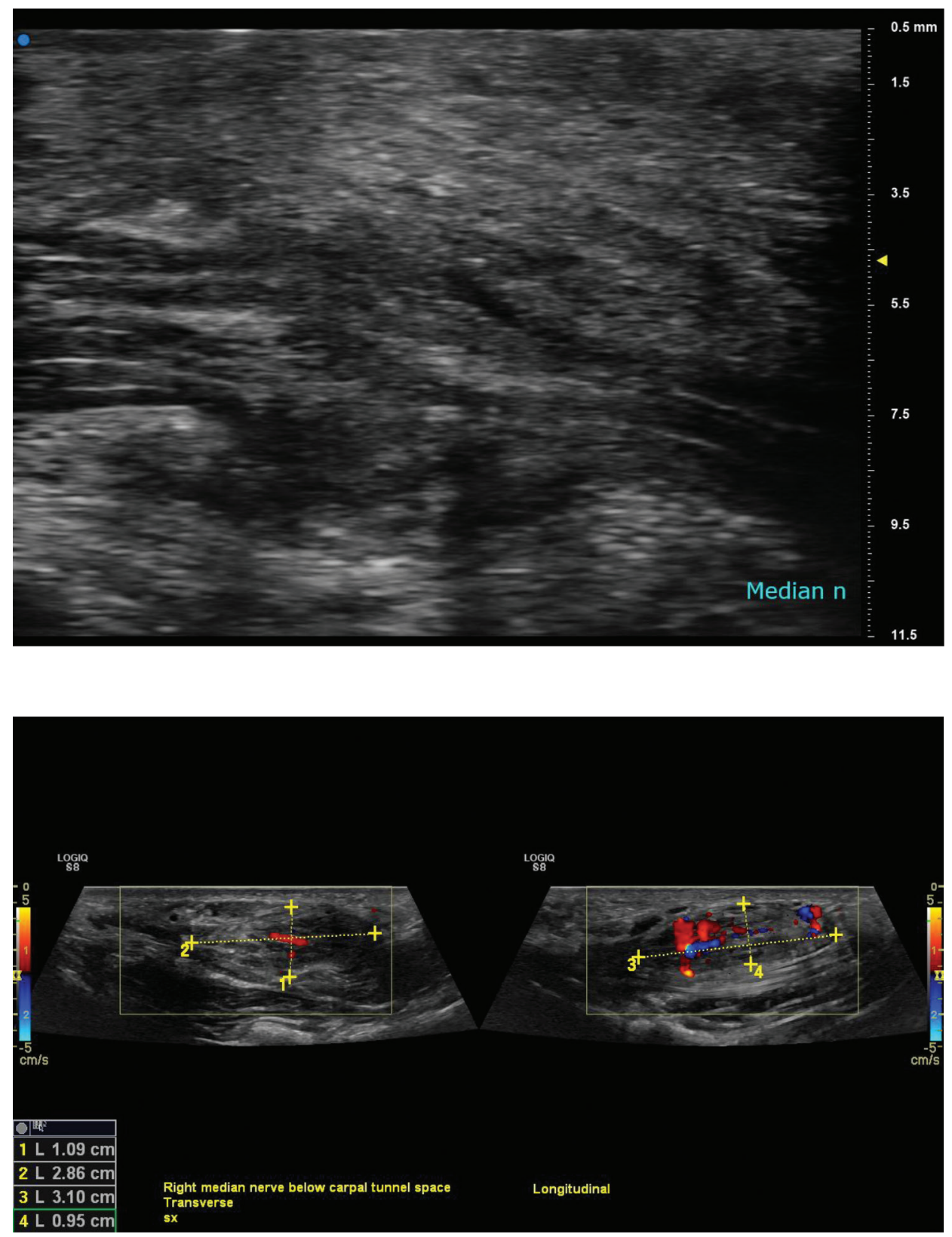

\section{FIGURE 4-29}

Sonograms of a short- and longaxis view of a vascular mass within the median nerve in the palm. The color Doppler flow is seen within the mass. 


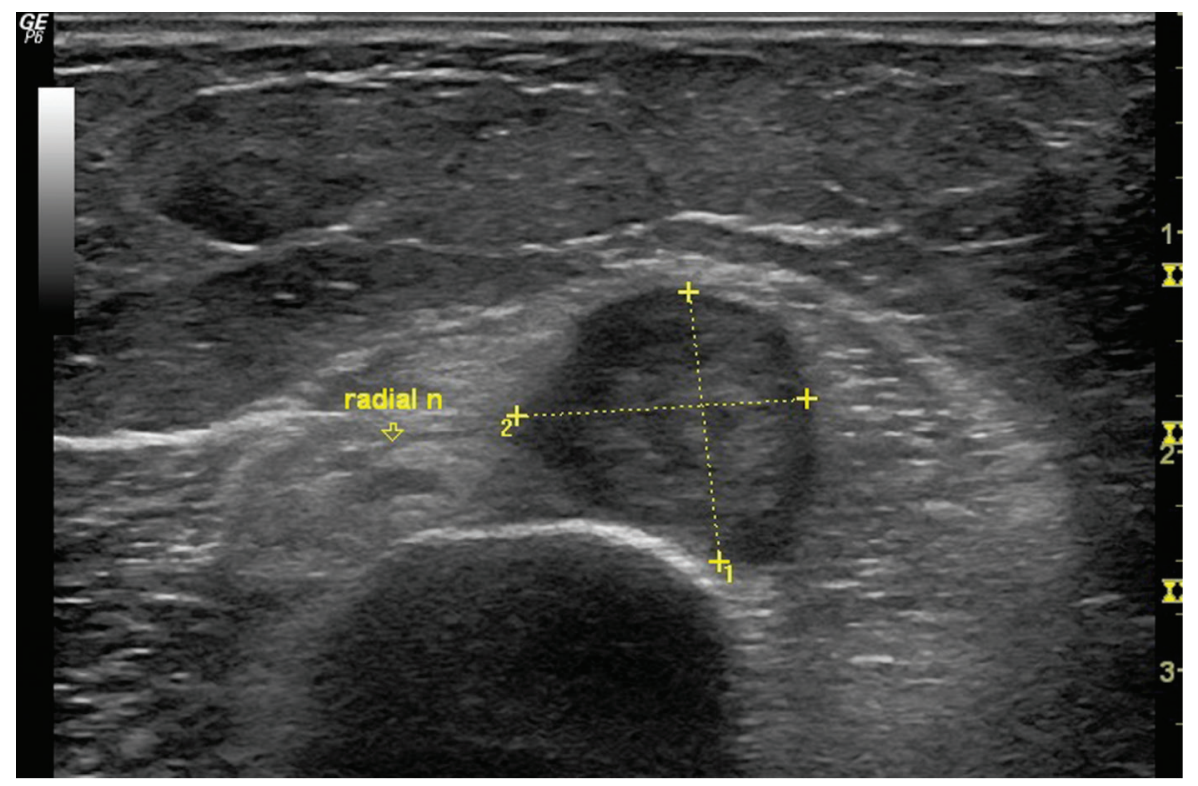

\section{FIGURE 4-30}

Sonogram of a mass near the radial nerve in the arm. Ultrasound is used to define the characteristics of the mass including echotexture, shape, and size. The mass should also be followed in both proximal and distal directions to demonstrate any relationship to the surrounding tissue, including nerve, muscle, and bone. the relation between the mass and other tissue structures, including the nerve (Chiou et al., 2003).

If the tumor is neurogenic, ultrasound can be used to identify the parent nerve and any plexiform elements. If nonneurogenic, determination should be made on whether it is extrinsic or intrinsic. Ultrasound also helps to differentiate between cystic and solid masses and outline the size and shape. It also is an ideal modality to guide needle biopsies to obtain a histologic sample of the tumor. A detailed discussion of all tumors that can affect peripheral nerves is beyond the scope of this book. Intraneural and extraneural ganglia, schwannomas, and neurofibromas are discussed.

\section{INTRANEURAL GANGLIA}

Intraneural ganglia are rare and occur most commonly at the knee and involve the fibular and tibial nerves from the articular branches to the tibialfibular joint (Leijten et al., 1992). They are also seen in the suprascapular nerve at the posterior shoulder and the ulnar nerve at the elbow, as well as the radial and median nerves. They often present as motor deficit with pain and paresthesia (Aprin et al., 2007). Sonography of intraneural ganglia appear as other ganglia, as an encapsulated, well-defined, multiloculated mass that is hypoechoic or anechoic and has posterior acoustic enhancement (Lang et al., 1994; Masciocchi et al., 1992). The nerve fascicles can sometimes be seen being displaced around the mass. Although the precise source of intraneural ganglia remains controversial, growing evidence suggests that they extend from the nearby joint space through the articular branches (Spinner et al., 2003, 2008). Articular branches are small and often difficult to appreciate with ultrasound under normal conditions. They can often be seen with enlargement from an intraneural ganglion, and knowing the location of the articular branch leads to appropriate interpretation of this finding.

\section{EXTRANEURAL GANGLIA}

Extraneural ganglia reside outside of the peripheral nerve and usually arise from another portion of the nearby joint (FIGURE 4-31). They can sometimes create neuropathy by mass effect in tight spaces. Ultrasound can often detect a communicating neck to the adjacent joint or tendon sheath. Other conditions that can have a similar appearance include tenosynovitis, seroma, abscess, and varicose veins. Ultrasound guidance can assist with decompression of the ganglion in real time. 
FIGURE 4-31

Sonogram of a ganglion cyst (blue arrow) arising from the radial-capitellar joint. Note the anechoic loculations and the neck connecting to the joint space.

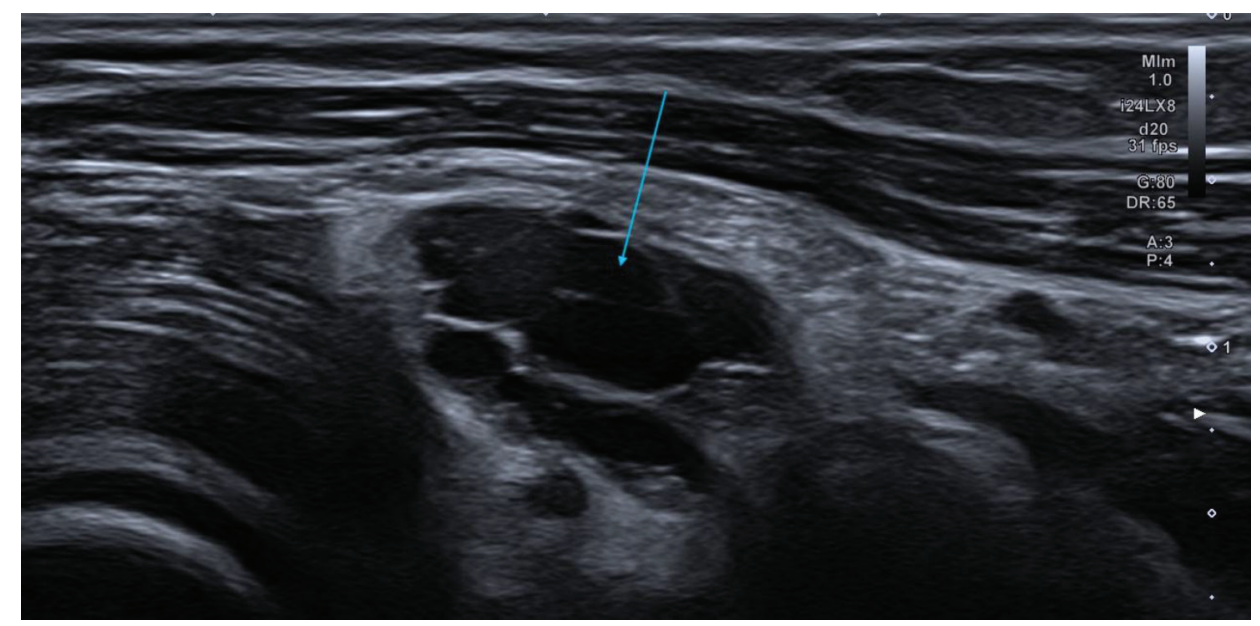

\section{PERIPHERAL NERVE SHEATH TUMORS}

\section{Schwannoma}

Schwannomas, also known as neurilemmomas, are benign intrinsic nerve tumors that are derived from the level of the nerve fascicle. They appear as a round globular tumor with speckled features (FIGURES 4-32). They typically present in middle age as a slowgrowing solitary lesion. Characteristic locations for schwannomas include nerve roots and major peripheral nerves. Schwannomas are more common than neurofibromas and have some characteristic differences (TABLE 4-1). The position of the schwannoma is usually eccentric to the nerve (Enzinger and Weiss, 1995). Schwannomas have a capsule and usually cavitation. Unlike localized neurofibromas, schwannomas are not usually associated with syndromes such as neurofibromatosis type 1 .

On ultrasound, schwannomas appear as wellcircumscribed solid soft-tissue masses with features as described earlier (Beggs, 1999). The nerve should be seen in continuity with the mass, and scanning should be done proximally and distally to identify the associated nerve. A schwannoma generally does not invade tissue but causes problems by mass effect.

\section{Neurofibromas}

Neurofibromas are much less common than schwannomas and are often associated with other syndromes such as neurofibromatosis type 1 . Neurofibromas are usually centered in the nerve tissue rather than eccentric to it as with schwannomas (FIGURE 4-33). Neurofibromas typically present in adolescence and do not have a capsule. They typically occur in groups. Plexiform neurofibromas should be identified when possible as they typically cannot be resected safely. Ultrasound has potential as a screening tool for identifying subclinical localized and plexiform neurofibromas in individuals with neurofibromatosis type 1 (Telleman et al., 2017).

\section{Other Variations of Schwannomas and Neurofibromas}

Other variations of schwannomas and neurofibromas exist. Hybrid tumors, although somewhat rare, can have schwannoma with neurofibroma (Sintzoff, Bank et al., 1992). Schwannomatosis, a condition with multiple lesions, can occur. Also plexiform schwannomas and neurofibromas can develop that are significantly more difficult to resect (Korf, 1999). All of these variations require sufficient suspicion and investigation to obtain the proper diagnosis and optimize treatment.

\section{Malignant Peripheral Nerve Sheath Tumors}

Malignant peripheral nerve sheath tumors often present with worsening pain, frequently described as lancinating, as well as progressive neurologic dysfunction. The symptoms and functional loss are often defined by their location. Malignant tumors should undergo biopsy for tissue diagnosis and staging 


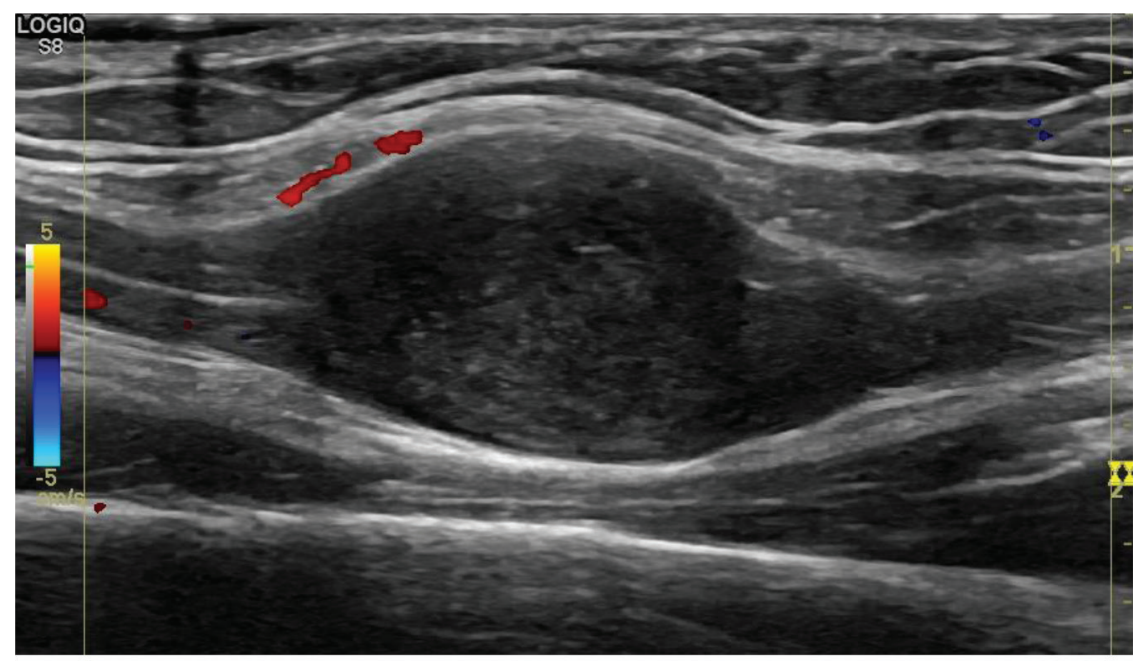

(A)

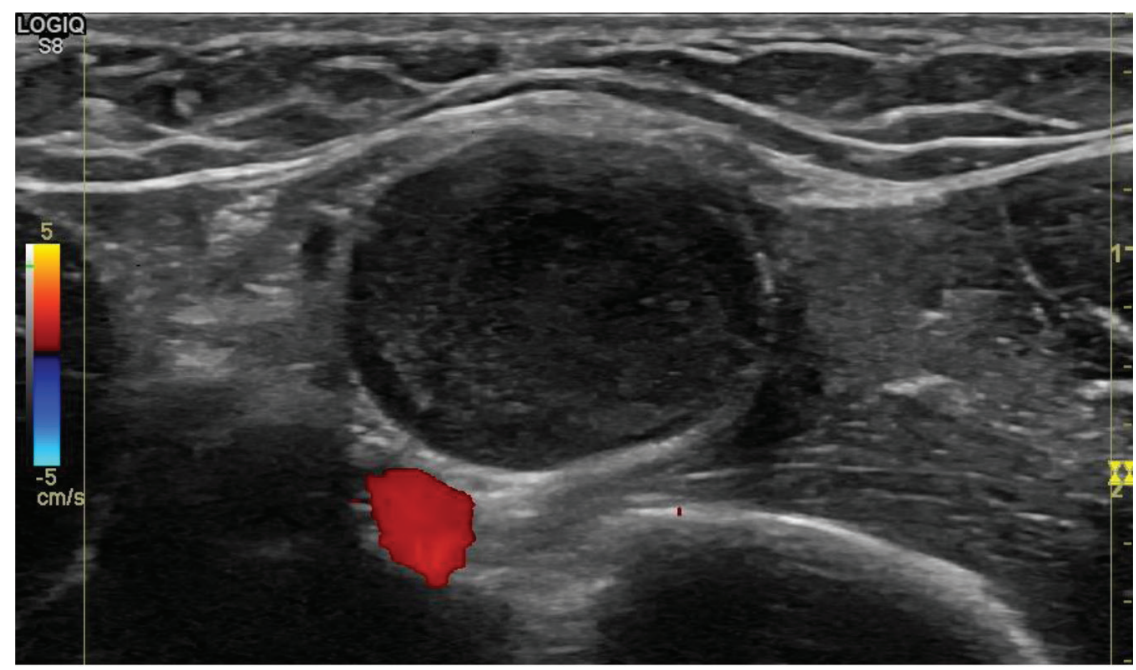

(B)

\section{FIGURE 4-32}

Sonograms of a schwannoma of the ulnar nerve in the arm. (A) A long-axis view that reveals the length of the tumor and helps confirm that it is contiguous with the nerve. It also illustrates the relative mass effect it has on the underlying triceps brachii muscle. (B) A short-axis view of the tumor provides additional visualization of surrounding tissue. It also compliments the long-axis view by providing the relative width.

TABLE 4-1

Distinguishing Schwannomas from Neurofibromas

\begin{tabular}{ll}
\hline SCHWANNOMAS & NEUROFIBROMAS \\
Nerve is eccentric to mass & Nerve through center of mass \\
Has a capsule & Does not have a capsule \\
Has cavitation & Rarely has cavitation \\
Not associated with NF1* & Localized type seen in $\mathrm{NF}^{*}$ \\
Malignant transformation: rare & Malignant transformation: $2 \%$ \\
Appear in adulthood & Appear in adolescence \\
Have more vascularity & Have less vascularity \\
\hline
\end{tabular}

$\mathrm{NF}$, neurofibromatosis.

*Neurofibromatosis type 1. 
FIGURE 4-33

Sonogram of neurofibromas in the median nerve. Note that the tumors are centrally located with respect to the nerve.

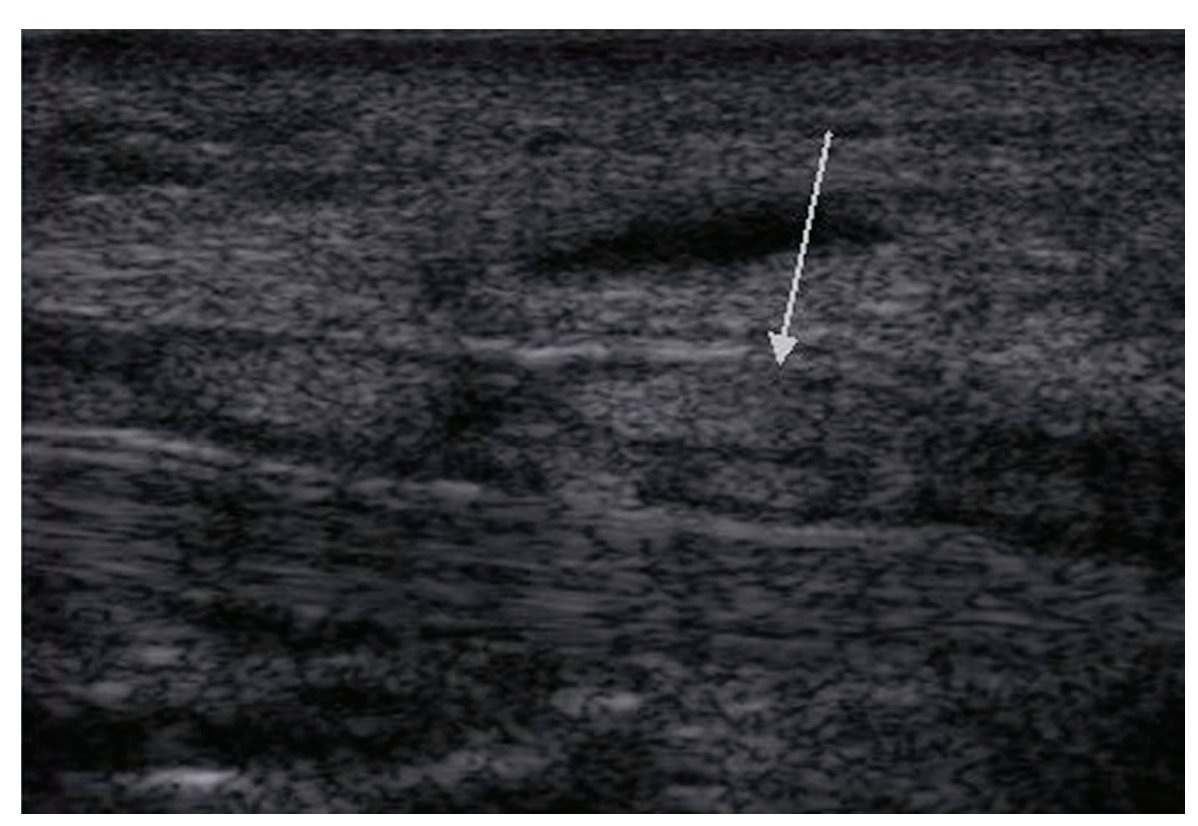

before surgical resection. Ultrasound is the ideal imaging modality for performing fine needle aspiration biopsy and larger biopsies.

\section{Other Tumors}

There are other types of neural tumors that also have to be considered. This includes perineuriomas (aka localized "hypertrophic neuropathy") and adipose tumors such as fibrolipomatous hamartoma (FIGURE 4-34) and macrodystrophia lipomatosa, as well as desmoid tumors, which are intermediate between benign and malignant (Gilet et al., 2008; Kim et al., 2005; Lusk et al., 1987).

The history and physical examination remain an important element in the assessment of a mass, and imaging should not be considered an adequate evaluation in isolation. Other conditions such as thrombosed vessels and enlarged lymph nodes can resemble nerve tumors (King et al., 1997). Clinical evidence to confirm the diagnosis should be sought when a nerve tumor is suspected. Imaging should be performed in more than one view for additional perspective. The shape, echotexture, size, and border should be detailed. The relative position of the tumor should be assessed, including its relationship to the nerve and surrounding vessels. Inspection for relative mass effect and any invasion into surrounding tissue should be performed. Doppler imaging should be used to identify relative vascularity of the mass as well as localized surrounding vessels. Additional imaging with MRI can provide further information in challenging cases. Ultimately biopsy or surgical removal for histopathology should be used when a definitive diagnosis is needed.

\section{ULTRASOUND-GUIDED NERVE BLOCKS}

Although a detailed discussion of nerve blocks is beyond the scope of this book, an increasing body of literature is developing regarding the use of ultrasound guidance for nerve blocks including perineural catheter placements (Dhir and Ganapathy, 2008; Ilfeld et al., 2010; Mariano et al., 2009, 2010; Neal and Chan, 2007; Niazi, 2009; Sandhu and Capan, 2002; Swenson et al., 2008). Chapter 15 discusses many of the considerations involved in the use of ultrasound for general needle guidance.

Most nerve blocks that use electrical stimulation for guidance of perineural catheter insertion advance the needle parallel to the longitudinal axis of the target nerve. By contrast, it is more conventional with ultrasound guidance to use a needle in-plane approach to the transducer and a perpendicular nerve-to-needle orientation. With ultrasound, crosssection or short-axis views allow for easier differentiation of the nerve from surrounding tissue. By maintaining in-plane orientation of the needle to 


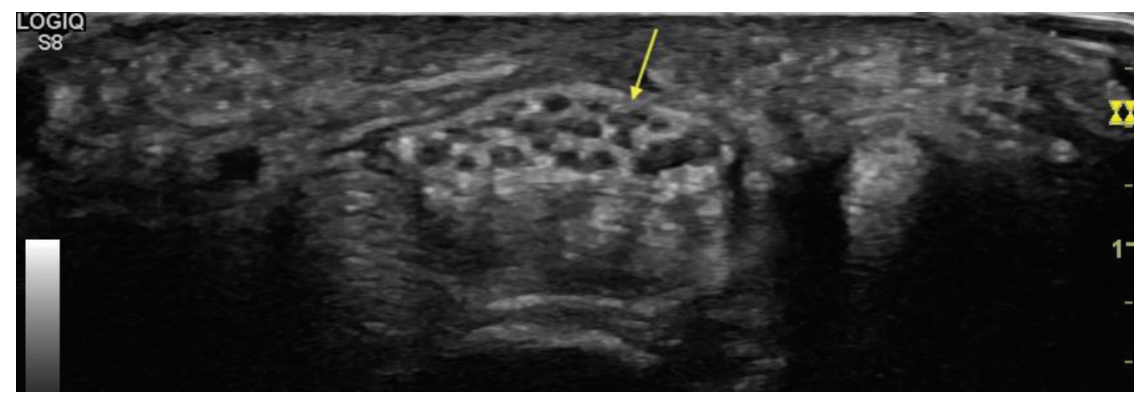

FIGURE 4-34

Sonogram of a fibrolipomatous hamartoma seen within the median nerve at the carpal tunnel. Note the dramatic enlargement of the entire nerve, crowding the carpal tunnel space, as well as hyperechoic fatty tissue surrounding the thickened hypoechoic fascicles.

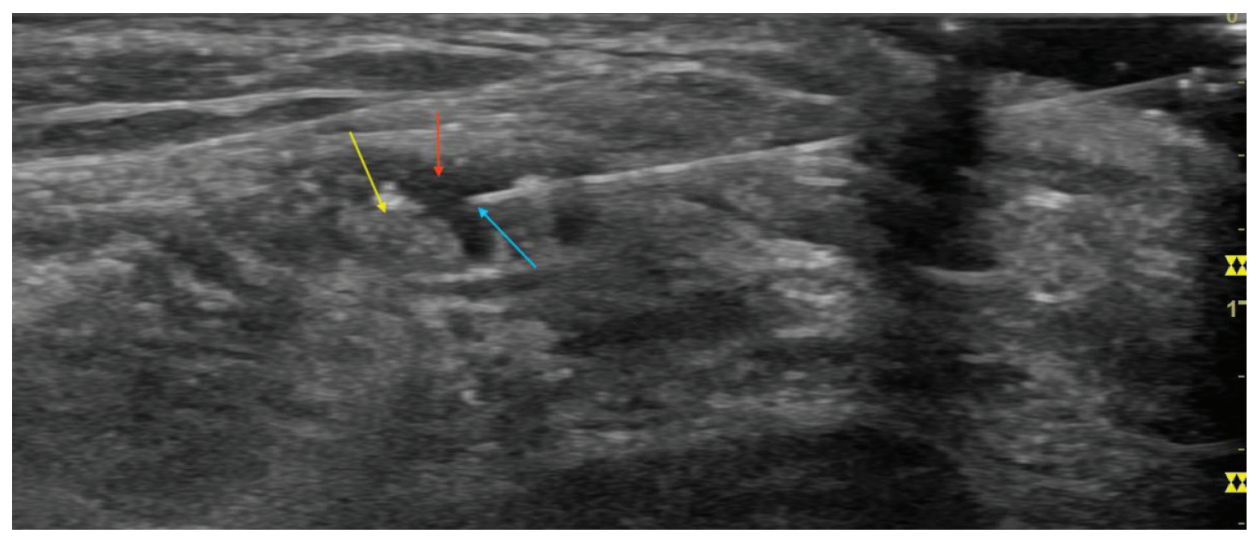

FIGURE 4-35

Sonogram of a lateral cutaneous nerve of the thigh block below the inguinal ligament. The needle tip (blue arrow) is seen near the lateral cutaneous nerve of the thigh (yellow arrow). The circumferential spread of the local anesthetic is also easily visualized (red arrow).

the transducer, real-time, needle-tip visualization and identification of its location relative to the target nerve is obtained. Additionally, the subsequent perineural spread of the local anesthetic injection can be visualized with evaluation of need for needle repositioning when necessary (Gray, 2006; FIGURE 4-35; ( VIDEO 4-12). Some authors advocate the use of ultrasound guidance for performing incremental injections to create circumferential spread around the nerve and increase the rate of sensory block (Brull et al., 2011). Ultrasound has been having an expanding role in interventional procedures of this nature and is an ideal modality for therapeutic injections and blocks of peripheral nerves (Strakowski, 2016).

\section{REFERENCES}

Please note, the full reference list for this chapter appears in the digital product found on http://connect.springerpub.com/content/ book/978-0-8261-7073-6/chapter/ch04 